\title{
A K-Theoretic \\ Relative Index Theorem and Callias-Type Dirac Operators
}

\section{U. Bunke}

SFB 288 Preprint No. 98

Diese Arbeit ist mit Unterstuitzung des von der Deutschen Forschungsgemeinschaft getragenen Sonderforschungsbereiches 288 entstanden und als Manuskript verviclfätigt worden.

Berlin, Dezember 1993 



\title{
A K-Theoretic Relative Index Theorem and Callias-Type Dirac Operators
}

\author{
Ulrich Bunke*
}

December 6, 1993

\begin{abstract}
We prove a relative index theorem for Dirac operators acting on an $A-C^{*}$ Clifford bundle, where $A$ is some $C^{*}$-algebra. We develop the index theory of complex and real Callias-type Dirac operators.
\end{abstract}

\section{Contents}

2 A K-theoretic relative index theorem 2

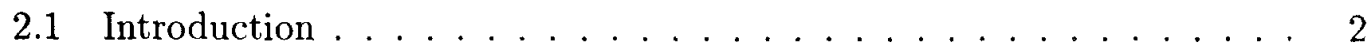

2.2 Analytical results . . . . . . . . . . . . . . . . 5

2.3 Cutting and pasting invariance ............. 13

2.4 Cobordisms of positive scalar curvature metrics . . . . . . . . . . 14

3 Index theory for Callias type operators 16

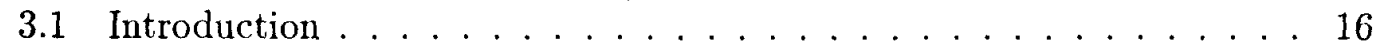

3.2 The Higson corona and admissible endomorphisms . . . . . . . . . . . 20

3.3 The odd-dimensional case . . . . . . . . . . . . . . 22

3.3 .1 The $\operatorname{map} u \ldots \ldots \ldots \ldots \ldots . \ldots \ldots 22$

3.3 .2 Computation of $u \ldots \ldots \ldots \ldots \ldots$

3.3.3 Interpretation as KK-intersection product . . . . . . . . 25

3.3.4 The spectral flow pairing $s \ldots \ldots \ldots . \ldots . \ldots 27$

3.4 The even-dimensional case . . . . . . . . . . . . . . . . . 28

3.4 .1 The construction of $g \ldots \ldots \ldots \ldots \ldots$

3.4 .2 Computation of $g \ldots \ldots \ldots \ldots \ldots$

3.4.3 Interpretation as $\mathbf{K K}$-intersection product . . . . . . . . . 30

*Institut für Reine Mathematik, Humboldt-Universität zu Berlin, Ziegelstr.13a, Berlin 10099 , Germany, E-mail: ubunke@mathematik.hu-berlin.de 
3.4 .4 The relative index pairing $r \ldots \ldots \ldots \ldots \ldots$

3.5 The boundary map in $\mathbf{K}$-homology . . . . . . . . . . . 33

3.6 Real Callias-type operators . . . . . . . . . . . . . . 35

\section{1}

This paper consists of two fairly independent sections. In the first section we consider the index theory of Dirac operators acting on $A-C^{*}$-Clifford bundles over complete Riemannian manifolds. Assuming invertibility at infinity we develop the analytical details for producing a Kasparov module representing the index of the Dirac operator. Our main result is the $\mathbf{K}$-theoretic relative index theorem. We provide applications related to the positive scalar curvature question.

In the second section we develop the index theory of Callias-type Dirac operators on odd as well as on even-dimensional manifolds in a systematic way. We show, how the construction of Callias type operators is related to the boundary map in the long $\mathbf{K}$-theory sequence of $\left(\bar{M}, \partial_{h} M\right) . M$ is a complete Riemannian manifold and $\bar{M}$ is a certain compactification by the Higson corona $\partial_{h} M$. We also consider real Dirac operators of Callias type being equivariant with respect to the action of some Clifford algebra. The resulting index theorem is again applied to obstructions against positive scalar curvature metrics.

This work was partly done at the Max Planck Institut für Mathematik, Bonn. The author wants to thank Stephan Stolz for the very stimulating discussion. He initiated this work by posing the problems, which are solved as applications of the $\mathbf{K}$-theoretic relative index theorem, and suggested many simplifications. There were also profitable discussions with S. Hurder. The author thanks the referees for forcing him to rewrite a miserable first version of that paper. In the meantime [2] appeared, it contains the index theorem for Callias-type operators on odd-dimensional manifolds.

\section{A K-theoretic relative index theorem}

\subsection{Introduction}

Let $D: C^{\infty}(M, E) \rightarrow C^{\infty}(M, E)$ be a generalized Dirac operator acting on sections of a $\mathbf{Z}_{2}$-graded Clifford bundle $E$ over a complete Riemannian manifold. If zero is not in the essential spectrum $\sigma_{e s s}(D)$ of $D$, then the index

$$
\text { ind } D=\operatorname{dim} \operatorname{ker} D^{+}-\operatorname{dim} \operatorname{ker} D^{-}
$$

is well-defined. We say that $D$ is positive at infinity if there is a constant $c>0$ and a compact set $K \subset M$ such that $R_{\mid M \backslash K} \geq c$, where $R:=D^{2}-\Delta$ is the endomorphism occurring in the Weitzenböck formula. If $D$ is positive at infinity, then $0 \notin \sigma_{\text {ess }}(D)$. 
The original version of the relative index theorem due to Gromov/Lawson [16] computes ind $D_{1}-$ ind $D_{2}$ for two Dirac operators that are positive at infinity and coincide outside of compact sets. Thus, the $D_{i}$ live on manifolds $M_{i}, i=1,2$, and there are open cocompact sets $U_{i} \subset M_{i}$ with smooth boundary $\partial \bar{U}_{1} \cong \partial \bar{U}_{2}$ such that $D_{1 \mid U_{1}} \cong D_{2 \mid U_{2}}$ and $R_{i \mid U_{i}} \geq c>0$. The equality $D_{1 \mid U_{1}} \cong D_{2 \mid U_{2}}$ means that there is an isometry $\gamma: U_{1} \rightarrow U_{2}$ covered by an isomorphism of Hermitian vector bundles $\Gamma: E_{1 \mid U_{1}} \rightarrow E_{2 \mid U_{2}}$ such that $D_{1} \Gamma^{*}=\Gamma^{*} D_{2}$. We deform the metric of $M_{1}$ and the Clifford bundle structure of $E_{i}$ near $\partial \bar{U}$ such that they become compatible with a product structure on a collar neighbourhood of $\partial \bar{U}$. Moreover, we consider $E_{2 \mid M_{2} \backslash U_{2}}$ with the opposite Clifford bundle structure, where $X \in T M_{2}$ acts by $-X$ and with the opposite grading. Let $M^{\sharp}:=M_{1} \backslash U_{1} U_{\partial \bar{U}} M_{2} \backslash U_{2}$ and glue the bundles using the morphism given by Clifford multiplication with the unit normal vector at $\partial \bar{U}_{1}$ (a nice reference for the relative index theorem, such glueing procedures and related topics is Booß/Wojciechowski [5]). Let $D^{\sharp}$ be the resulting Dirac operator over $M^{\sharp}$.

\section{Theorem 2.1 (Gromov/Lawson)}

$$
\text { ind } D_{1}-\text { ind } D_{2}=\text { ind } D^{\sharp} \text {. }
$$

Another way to look upon this theorem is as follows. Consider $M=M_{1} \cup M_{2}$ and the opposite Clifford bundle structure and grading on $E_{2}$. Let $D$ be the Dirac operator over $M$. Obviously ind $D=$ ind $D_{1}-i n d D_{2}$. We can cut $M$ at $\partial \bar{U}_{1} \cup \partial \bar{U}_{2}$ and glue it together again using the diffeomorphism interchanging the two boundary components, obtaining $\tilde{M}$ together with a new $\mathbf{Z}_{2}$-graded Clifford bundle and a Dirac operator $\tilde{D}$. Then $\tilde{M}=M^{\sharp} \cup\left(U_{1} \cup_{\partial U} U_{2}\right)$ and $\tilde{D}$ is invertible over $U_{1} \cup_{\partial U} U_{2}$. Hence ind $\tilde{D}=$ ind $D^{\sharp}$. The relative index theorem states that cutting and glueing as described above does not change the index:

$$
\text { ind } D=\operatorname{ind} \tilde{D} \text {. }
$$

There are several generalizations of the relative index theorem [8],[10], Donnelly [15], Anghel [1],[2], Brüning/Moscovici [7], Borisov/Müller/Schrader [6], Roe [26]. The aim of the present section is to give a $\mathbf{K}$-theoretic variant of this theorem, which applies also for operators acting on $A-C^{*}$-Clifford bundles, where $A$ is a $C^{*}$-algebra over the base field $k=\mathbf{R}, \mathbf{C}$. Such operators were considered first by Miščenko/Fomenko [24].

Let $M$ be a complete Riemannian manifold and $A$ be a $\mathbf{Z}_{2}$-graded $C^{*}$-algebra. An $A-C^{*}$-Clifford bundle $E$ is a bundle of projective graded A-Hilbert- $C^{*}$-modules together with a metric connection and a Clifford multiplication satisfying the Leibnitz rule and the compatibility with the scalar products of the fibres. If the $C^{*}$-algebra $A$ is not unital, then, by convention, a module $V$ over $A$ is called projective if $1 \in B(V)$ is compact (See Blackadar [4] for the definition of compact operators over A-Hilbert$C^{*}$-modules). Let $D$ be the associated Dirac operator. We define Sobolev spaces $H^{l}, l \geq 0$, (see Fomenko/Miščenko [24] and (2)) which are A-Hilbert- $C^{*}$-modules. We have $D \in B\left(H^{l}, H^{l-1}\right), l \geq 1$. Our basic assumption is 
Assumption 1 (invertibility at infinity) There is a function $f \in C_{c}^{\infty}(M)$ such that $f \geq 0$ and $\left(D^{2}+f\right)^{-1} \in B\left(H^{0}, H^{2}\right)$ exists.

We can then construct a Kasparov module (see Blackadar [4] for definitions) representing the index of $D$. In Lemma 2.8 we will give sense to the operator $F:=$ $D\left(D^{2}+f\right)^{-1 / 2} \in B\left(H^{0}\right)^{\text {odd }}$. Let $C_{g}^{\infty}(M)$ be the algebra of bounded smooth $k$-valued functions with a gradient vanishing at infinity and let $C_{g}(M)$ be the $C^{*}$-algebra closure of $C_{g}^{\infty}(M)$ with respect to the supremum norm. There is a *-homomorphism $C_{g}(M) \rightarrow B\left(H^{0}\right)$ given by multiplication. $\left(H^{0}, F\right)$ is a Kasparov module over the pair of $C^{*}$-algebras $\left(C_{g}(M), A\right)$ by Proposition 2.13. Let the symbol $M$ stand for all structures over $M$ and $[M] \in \mathrm{KK}\left(C_{g}(M), A\right)$ be the class represented by $\left(H^{0}, F\right)$. By Lemma 2.10 the class $[M]$ does not depend on the choice of the function $f \in C_{c}^{\infty}(M)$.

The reason for using the rather complicated notions of Kasparovs KK-Theory is that we want to apply the relative index theorem in the case of graded $C^{*}$-algebras. Until now, a reasonable extension of the index theory of Miščenko/Fomenko to graded algebras has not been developed. In this section we will use only the definition of the KK-groups and the equivalence relations 'compact perturbation' and 'operator homotopy'. Thus, nothing really deep is involved.

We will prove the following relative index theorem. Let $E_{i} \rightarrow M_{i}, i=1,2$, be two $A-C^{*}$-Clifford bundles with Dirac operators $D_{i}$ being invertible at infinity. Let $W_{i} \cup_{N_{i}} V_{i}$ be partitions of $M_{i}$ where $N_{i}$ are compact hypersurfaces. Assume that there is a commutative diagram of isomorphisms of all structures

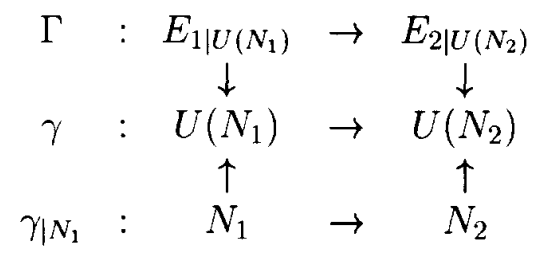

where the $U\left(N_{i}\right)$ are tubular neighbourhoods of $N_{i}, i=1,2$. We cut $M_{i}$ at $N_{i}$, glue the pieces together interchanging the boundary components and obtain $M_{3}:=$ $W_{1} \cup_{N} V_{2}$ and $M_{4}:=W_{2} \cup_{N} V_{1}$. Moreover, we glue the bundles using $\Gamma$, which yields $A$ - $C^{*}$-Clifford bundles $E_{3} \rightarrow M_{3}$ and $E_{4} \rightarrow M_{4}$ and we assume that $D_{i}, i=3,4$ are again invertible at infinity. We define $\left[M_{3}\right]$ and $\left[M_{4}\right]$ as above.

The algebra $C_{g}(M)$ is unital. Hence, there is an embedding $i: k \rightarrow C_{g}(M)$ and an induced map

$$
i^{*}: \mathbf{K K}\left(C_{g}(M), A\right) \rightarrow \mathbf{K K}(k, A) .
$$

Set $\left\{M_{i}\right\}:=i^{*}\left[M_{i}\right] \in \mathbf{K K}(k, A)$ for $i=1, \ldots, 4$.

Theorem 2.2 (K-theoretic relative index theorem)

$$
\left\{M_{1}\right\}+\left\{M_{2}\right\}-\left\{M_{3}\right\}-\left\{M_{4}\right\}=0
$$


In the present section we consider those applications which are the motivation for proving a K-theoretic relative index theorem. Let $k:=\mathbf{R}, M^{n}$ be complete, Riemannian and spin, $E$ be the real Clifford bundle with fibres isomorphic to the Clifford algebra $C^{n, 0}$ admitting a right $C^{n, 0}$-action and $V$ be a flat bundle of A-Hilbert- $C^{*}$ modules over $M$. Define the $C^{n, 0} \otimes A-C^{*}$-Clifford bundle $S:=E \otimes V$. Assume that there is a compact set $K \subset M$ and a constant $c>0$ such that for the scalar curvature $s$ we have the estimate $s_{\mid M \backslash K} \geq c$. We show that under some condition of bounded geometry on $V$ the operator $D$ is invertible at infinity (see Lemma 2.18).

We solve two problems suggested to the author by S. Stolz. The first asserts that cutting and pasting at compact hypersurfaces with positive scalar curvature does not change the index of $D$ (Theorem 2.16). The second problem is to construct, for any discrete group $\pi$, a group homomorphism

$$
R_{n}(\pi) \rightarrow \mathbf{K K}_{n}\left(\mathbf{R}, C_{r}^{*}(\pi)\right),
$$

where $R_{n}(\pi)$ is a group of $n$-dimensional $B=B \pi \times B$ Spin manifolds with prescribed positive scalar curvature metric at the boundary $\partial M$.

In view of the applications in the second section note that all the results of the present section remain true if one replaces the Dirac operator associated to the $A-C^{*}$-Clifford bundle by an $A$-equivariant Callias-type operator.

\subsection{Analytical results}

Let $M$ be a complete Riemannian manifold and $E$ be an $A-C^{*}$-Clifford bundle with associated Dirac operator $D$. We form the completions $H^{l}, l \geq 0$, of $C_{c}^{\infty}(M, E)$ with respect to the norms

$$
\|\phi\|_{l}^{2}=\sum_{k=0}^{l} \int_{M}\left\|D^{k} \phi(x)\right\|^{2}, \quad \phi \in C_{c}^{\infty}(M, E),
$$

where the norm on the right-hand side is the pointwise norm coming from the $A$ Hilbert- $C^{*}$-module structure of the fibres. The $H^{l}$ are A-Hilbert- $C^{*}$-modules with the $A$-valued scalar product

$$
<\phi, \psi>_{l}=\sum_{k=0}^{l} \int_{M}<D^{k} \phi(x), D^{k} \psi(x)>.
$$

There is an analogue of Rellich's theorem.

Lemma 2.3 (Miščenko/Fomenko,[24]) For any $f \in C_{c}^{\infty}(M)$ the multiplication operator $f: H^{l} \rightarrow H^{k}$ is compact for $k<l$.

By continuity and the closedness of the space of compact operators the compactness of the multiplication operators with functions will extend to larger classes of functions. $D$ extends to an operator $D \in B\left(H^{l}, H^{l-1}\right), \forall l \geq 1$. The symmetry property of $D$ extends to $H^{1}$ :

$$
<\psi, D \phi>=<D \psi, \phi>, \quad \psi, \phi \in H^{1}
$$


In the present subsection we carry out certain calculations which, at a first glance, may appear as trivial applications of the spectral theory of self-adjoint operators. However, one should be careful since no such theory has been developed for unbounded self-adjoint operators over an $A$-Hilbert- $C^{*}$ module. We will give fairly detailed arguments for the first few lemmas. The estimates and compactness claimed in the later proofs follow by similar arguments using suitable factorizations.

Let $D$ be invertible at infinity and set $B:=\left(D^{2}+f\right)^{-1}$.

Lemma 2.4 We have

$$
0<d:=i n f_{\psi \in H^{2},\|\psi\|_{0}=1}\left(\|D \psi\|_{0}^{2}+\|\sqrt{f} \psi\|_{0}^{2}\right) .
$$

Proof: Let $\psi \in H^{2}$ with $\|\psi\|_{0}=1$. Then

$$
\begin{aligned}
1 & =\left\|<\psi, \psi>_{0}\right\| \\
& =\left\|<\left(D^{2}+f\right) B \psi, \psi>_{0}\right\| \\
& =\left\|<D B \psi, D \psi>_{0}+<\sqrt{f} B \psi, \sqrt{f} \psi>_{0}\right\| \\
& \leq\|D B\|_{B\left(H^{0}\right)}\|D \psi\|_{0}+\sup _{x \in M}|\sqrt{f(x)}|\|B\|_{B\left(H^{0}\right)}\|\sqrt{f} \psi\|_{0} \\
& \leq C\left(\|D \psi\|_{0}^{2}+\|\sqrt{f} \psi\|_{0}^{2}\right)^{1 / 2}
\end{aligned}
$$

for some $C<\infty$. The lemma follows with $d \geq C^{-2}$.

Lemma 2.5 For $\lambda \geq 0$ the operator

$$
R(\lambda):=\left(D^{2}+f+\lambda^{2}\right)^{-1} \in B\left(H^{0}, H^{2}\right)
$$

exists and satisfies the estimates

$$
\begin{aligned}
\|R(\lambda)\|_{B\left(H^{0}\right)} & \leq\left(d+\lambda^{2}\right)^{-1} \\
\left\|D^{2} R(\lambda)\right\|_{B\left(H^{0}\right)} & \leq C
\end{aligned}
$$

with $C<\infty$ independent of $\lambda$.

Proof: Assume that the lemma holds for all $0 \leq \lambda \leq \Lambda$. This is certainly true for $\Lambda=0$. Then, for all $\lambda$ with $\left|\lambda^{2}-\Lambda^{2}\right|<\left(d+\Lambda^{2}\right)$,

$$
R(\lambda)=\sum_{i=0}^{\infty} R(\Lambda)^{i}\left(\Lambda^{2}-\lambda^{2}\right)^{i} R(\Lambda) \in B\left(H^{0}, H^{2}\right)
$$

The estimate (3) follows from

$$
\left\|\left(D^{2}+f+\lambda^{2}\right) \psi\right\|_{0} \geq\left(d+\lambda^{2}\right)\|\psi\|_{0}, \quad \forall \psi \in H^{2}
$$

(see Lemma 2.4) since $H^{2} \subset H^{0}$ is dense. (4) follows from (3) by

$$
\left\|D^{2} R(\lambda) \phi\right\|_{0} \leq\|\phi\|_{0}+\left\|\left(f+\lambda^{2}\right) R(\lambda) \phi\right\|_{0} \leq C\|\phi\|_{0}, \quad \forall \phi \in H^{0} .
$$


Lemma 2.6 We have $D R(\lambda) \in B\left(H^{0}\right)$ and the estimate

$$
\|D R(\lambda)\|_{B\left(H^{0}\right)} \leq C\left(d+\lambda^{2}\right)^{-1 / 2}
$$

holds for some $C<\infty$ independent of $\lambda \geq 0$.

Proof: There is a $C<\infty$ such that for all $\phi \in H^{0}$ with $\|\phi\|_{0}=1$

$$
\begin{aligned}
\|D R(\lambda) \phi\|_{H^{0}}^{2} & =\|<D R(\lambda) \phi, D R(\lambda) \phi>\|_{0} \\
& =\left\|<R(\lambda) \phi, D^{2} R(\lambda) \phi>\right\|_{0} \\
& \leq\|R(\lambda) \phi\|_{0}\left\|D^{2} R(\lambda) \phi\right\|_{0} \\
& \leq C^{2}(d+\lambda)^{-1}
\end{aligned}
$$

holds by $(3),(4)$. Thus, $D R(\lambda)$ is bounded and satisfies the desired estimate. We have to verify that it has an adjoint. Formally, $(D R(\lambda))^{*}=R(\lambda) D$, but this does not make sense since $D$ is not defined on all $H^{0}$. First we check that $R(\lambda)^{*}=R(\lambda)$. For all $\phi \in H^{2}, \psi \in H^{0}$

$$
\begin{aligned}
<R(\lambda)\left(D^{2}+f+\lambda^{2}\right) \phi, \psi>_{0} & -<\left(D^{2}+f+\lambda^{2}\right) \phi, R(\lambda) \psi>_{0} \\
& =<\phi, \psi>_{0}-<\phi,\left(D^{2}+f+\lambda^{2}\right) R(\lambda) \psi>_{0} \\
& =0
\end{aligned}
$$

using the symmetry of $D$. Any $\rho \in H^{0}$ has the representation $\rho=\left(D^{2}+f+\lambda^{2}\right) \phi$ for some $\phi \in H^{2}$. Thus, for any $\rho, \psi$

$$
<R(\lambda) \rho, \psi>=<\rho, R(\lambda) \psi>
$$

and hence $R(\lambda)^{*}=R(\lambda)$. Next we show

$$
(D R(\lambda))^{*}=D R(\lambda)+R(\lambda) \operatorname{grad}(f) R(\lambda)
$$

where $\operatorname{grad}(f)$ is the Clifford multiplication by the gradient vector field of $f$. For all $\psi, \phi \in H^{0}$ we have

$$
\begin{aligned}
<\psi, D R(\lambda) \phi>_{0}= & <\left(D^{2}+f+\lambda^{2}\right) R(\lambda) \psi, D R(\lambda) \phi>_{0} \\
= & <D^{2} R(\lambda) \psi, D R(\lambda) \phi>_{0}+<\left(f+\lambda^{2}\right) R(\lambda) \psi, D R(\lambda) \phi>_{0} \\
= & <D R(\lambda) \psi, D^{2} R(\lambda) \phi>_{0}+<R(\lambda) \psi,\left(f+\lambda^{2}\right) D R(\lambda) \phi>_{0} \\
= & <D R(\lambda) \psi, \phi>_{0}-<D R(\lambda) \psi,\left(f+\lambda^{2}\right) R(\lambda) \phi>_{0} \\
& +<R(\lambda) \psi,\left(f+\lambda^{2}\right) D R(\lambda) \phi>_{0} \\
= & <D R(\lambda) \psi, \phi>_{0}-<R(\lambda) \psi,\left[D,\left(f+\lambda^{2}\right)\right] R(\lambda) \phi>_{0} \\
= & <(D R(\lambda)+R(\lambda) \operatorname{grad}(f) R(\lambda)) \psi, \phi>_{0} .
\end{aligned}
$$

This shows the claim. 
Lemma 2.7 For any $\psi \in H^{1}$

$$
[D, R(\lambda)] \psi=-R(\lambda) \operatorname{grad}(f) R(\lambda) \psi .
$$

Proof: For all $\psi, \phi \in H^{1}$ we have by Lemma 2.6

$$
\begin{aligned}
<\psi, R(\lambda) D \phi>_{0} & =<D R(\lambda) \psi, \phi>_{0} \\
& =<\psi,(D R(\lambda)+R(\lambda) \operatorname{grad}(\lambda) R(\lambda)) \phi>_{0} .
\end{aligned}
$$

Lemma 2.8 For any $\psi \in H^{1}$ the integral

$$
F \psi:=\frac{2}{\pi} \int_{0}^{\infty} D R(\lambda) \psi d \lambda
$$

converges in norm and defines an operator $F \in B\left(H^{0}\right)^{\text {odd }}$.

Proof: For $\lambda \geq 0$

$$
R(\lambda)=\frac{1}{\lambda^{2}} B\left(B+1 / \lambda^{2}\right)^{-1},
$$

as it can be seen by composing with $D^{2}+f+\lambda^{2}$. Applying the functional calculus for bounded self-adjoint operators in the $C^{*}$-algebra $B\left(H^{0}\right)$ we have

$$
\begin{aligned}
\frac{2}{\pi} \int_{0}^{\infty} R(\lambda) d \lambda & =\frac{2}{\pi} \int_{0}^{\infty} B\left(B+1 / \lambda^{2}\right)^{-1} \frac{d \lambda}{\lambda^{2}} \\
& =\frac{2}{\pi} \int_{0}^{\infty} B\left(B+z^{2}\right) d z \\
& =B^{1 / 2},
\end{aligned}
$$

where the integrals converge in norm. There is a $C<\infty$ such that, for any $\psi \in H^{1}$, it holds

$$
\begin{aligned}
\left\|B^{1 / 2} D \psi\right\|_{0}^{2} & =\left\|<B^{1 / 2} D \psi, B^{1 / 2} D \psi>_{0}\right\| \\
& =\left\|<B D \psi, D \psi>_{0}\right\| \\
& =\left\|<(D B+B \operatorname{grad}(f) B) \psi, D \psi>_{0}\right\| \\
& =\left\|<D^{2} B \psi, \psi>_{0}+<D B \operatorname{Drad}(f) B \psi, \psi>_{0}\right\| \\
& \leq C\|\psi\|_{0}^{2} .
\end{aligned}
$$

$B^{1 / 2} D$ extends by continuity to a bounded operator $L: H^{0} \rightarrow H^{0}$. By Lemma 2.7 we can write

$$
F \psi:=L \psi-\frac{2}{\pi} \int_{0}^{\infty} R(\lambda) \operatorname{grad}(f) R(\lambda) d \lambda \psi
$$


The integral on the r.h.s is operator norm convergent by (3) and defines a bounded operator in $B\left(H^{0}\right)$. It remains to show that $L \in B\left(H^{0}\right)$, i.e. that $L$ admits an adjoint. For any $\phi \in H^{1}, \psi \in H^{2}$ it holds

$$
\begin{aligned}
<L \phi, \psi>_{0} & =<B^{1 / 2} D \phi, \psi>_{0} \\
& =<D \phi, B B^{1 / 2}\left(D^{2}+f\right) \psi>_{0} \\
& =<\phi, D B B^{1 / 2}\left(D^{2}+f\right) \psi>_{0} .
\end{aligned}
$$

The operator $D B B^{1 / 2}\left(D^{2}+f\right): H^{2} \rightarrow H^{0}$ satisfies

$$
\|\left(D B B^{1 / 2}\left(D^{2}+f\right) \psi\left\|_{0} \leq\right\| L\left\|_{B\left(H^{0}\right)}\right\| \psi \|_{0}\right.
$$

for all $\psi \in H^{2}$ and extends by continuity to $L^{*}: H^{0} \rightarrow H^{0}$. Hence, $L \in B\left(H^{0}\right)$.

Lemma $2.9 F-F^{*} \in K\left(H^{0}\right)$

Proof: By Lemma 2.8 and 2.7, for all $\psi \in H^{1}$,

$$
\left(F^{*}-F\right) \psi=\frac{2}{\pi} \int_{0}^{\infty} R(\lambda) \operatorname{grad}(f) R(\lambda) \psi d \lambda .
$$

By Lemma 2.5

$$
\|R(\lambda) \operatorname{grad}(f) R(\lambda)\|_{B\left(H^{0}\right)} \leq C\left(d+\lambda^{2}\right)^{-2}
$$

for some $C<\infty$ independent of $\lambda$. In order to see that the integrand is compact use Lemma 2.3 and the factorization.

$$
H^{0} \stackrel{R(\lambda)}{\longrightarrow} H^{2} \stackrel{\operatorname{grad}(f)}{\longrightarrow} H^{0} \stackrel{R(\lambda)}{\longrightarrow} H^{0},
$$

where the middle arrow is compact. A norm convergent integral of compact operators is compact by the closedness of the ideal of compact operators.

Lemma 2.10 Let $f, f_{1} \in C_{c}^{\infty}(M), f, f_{1} \geq 0$, be such that

$$
\left(D^{2}+f\right)^{-1},\left(D^{2}+f_{1}\right)^{-1} \in B\left(H^{0}, H^{2}\right)
$$

exist. Define $F, F_{1}$ using $f, f_{1}$. Then $F-F_{1} \in K\left(H^{0}\right)$.

Proof: We claim

$$
D R(\lambda)-D R_{1}(\lambda) \in K\left(H^{0}\right)
$$

and

$$
\left\|D R(\lambda)-D R_{1}(\lambda)\right\|_{B\left(H^{0}\right)} \leq C\left(d+\lambda^{2}\right)^{-3 / 2}
$$

for some $C<\infty$ independent of $\lambda$. The Lemma follows by integration. We have

$$
R(\lambda)-R_{1}(\lambda)=R(\lambda)\left(f_{1}-f\right) R_{1}(\lambda)
$$


as it can be seen by composing with $D^{2}+f+\lambda^{2}$. Property (7) follows from Lemma 2.3 and the factorization

$$
H^{0} \stackrel{R_{1}(\lambda)}{\longrightarrow} H^{2} \stackrel{f_{1}-f}{\longrightarrow} H^{0} \stackrel{D R(\lambda)}{\longrightarrow} H^{0}
$$

where the middle arrow is compact. By (3) and (4) we have

$$
\begin{aligned}
\left\|D R(\lambda)-D R_{1}(\lambda)\right\|_{B\left(H^{0}\right)} & \leq\|D R(\lambda)\|_{B\left(H^{0}\right)} \sup _{x \in M} \mid f_{1}(x)-f(x)\|\| R_{1}(\lambda) \|_{B\left(H^{0}\right)} \\
& \leq C\left(d+\lambda^{2}\right)^{-3 / 2}
\end{aligned}
$$

for some $C<\infty$ independent of $\lambda$.

Lemma $2.11 F^{2}-1 \in K\left(H^{0}\right)$

Proof: By (5), the compactness of the operator defined by the integral on the r.h.s of (6) and Lemma 2.9 it is enough to show that $L L^{*}-1 \in K\left(H^{0}\right)$. For $\psi \in H^{2}$ we have

$$
\begin{aligned}
\left(L L^{*}-1\right) \psi & =B^{1 / 2} D^{2} B B^{1 / 2}\left(D^{2}+f\right) \psi-\psi \\
& =B\left(D^{2}+f\right) \psi-\psi-B^{1 / 2} f B B^{1 / 2}\left(D^{2}+f\right) \psi \\
& =-B^{1 / 2} f B^{1 / 2} \psi
\end{aligned}
$$

Let " " denote equality modulo compact operators.

$$
\begin{aligned}
f B^{1 / 2} & =\frac{2}{\pi} \int_{0}^{\infty} f R(\lambda) d \lambda \\
& =\frac{2}{\pi} \int_{0}^{\infty}(R(\lambda) f+R(\lambda)(D \operatorname{grad}(f)+\operatorname{grad}(f) D) R(\lambda)) d \lambda \\
& \sim B^{1 / 2} f
\end{aligned}
$$

Hence, $L L^{*}-1 \sim-f B \in K\left(H^{0}\right)$ by Lemma 2.3 .

Lemma 2.12 Let $h \in C_{g}(M)$. Then $[F, h] \in K\left(H^{0}\right)$.

Proof: $h$ can be approximated in the supremum norm by functions $h_{n} \in C_{g}^{\infty}(M)$. Thus, it is enough to show the Lemma for $h \in C_{g}^{\infty}(M)$. Using Lemma 2.8 for any $\psi \in H^{1}$

$$
\begin{aligned}
{[F, h] \psi } & =\frac{2}{\pi} \int_{0}^{\infty}[D R(\lambda), h] \psi d \lambda \\
& =\frac{2}{\pi} \int_{0}^{\infty}(D[R(\lambda), h] \psi+\operatorname{grad}(h) R(\lambda) \psi) d \lambda
\end{aligned}
$$


where we employ that $h: H^{1} \rightarrow H^{1}$ is bounded. It takes some trouble to write down expressions which, in order to be well-defined, do not involve bounds on the second derivatives of $h$. It holds

$$
\begin{aligned}
{[R(\lambda), h]=} & R(\lambda) h-h R(\lambda) \\
= & R(\lambda) h\left(D^{2}+f+\lambda^{2}\right) R(\lambda)-D^{2} R(\lambda) h R(\lambda)-\left(f+\lambda^{2}\right) R(\lambda) h R(\lambda) \\
D^{2} R(\lambda) h R(\lambda)= & D R(\lambda) D h R(\lambda)-D R(\lambda) \operatorname{grad}(f) R(\lambda) h R(\lambda) \\
D[R(\lambda), h]= & D R(\lambda) h\left(D^{2}+f+\lambda^{2}\right) R(\lambda)-D^{2} R(\lambda) D h R(\lambda) \\
& -D^{2} R(\lambda) \operatorname{grad}(f) R(\lambda) h R(\lambda)-D\left(f+\lambda^{2}\right) R(\lambda) h R(\lambda) .
\end{aligned}
$$

We drop all terms that are integrable and compact and obtain the remainder

$$
\begin{aligned}
S(\lambda):= & D R(\lambda) h D^{2} R(\lambda)-D^{2} R(\lambda) D h R(\lambda) \\
= & D R(\lambda) h D^{2} R(\lambda)-D^{2} R(\lambda) h D R(\lambda)-D^{2} R(\lambda) \operatorname{grad}(h) R(\lambda) \\
= & D R(\lambda) h D^{2} R(\lambda)-D R(\lambda) D h D R(\lambda) \\
& +D R(\lambda) \operatorname{grad}(f) R(\lambda) h D R(\lambda)-D^{2} R(\lambda) \operatorname{grad}(h) R(\lambda) \\
= & -D R(\lambda) \operatorname{grad}(h) D R(\lambda)+D R(\lambda) \operatorname{grad}(f) R(\lambda) h D R(\lambda)-D^{2} R(\lambda) \operatorname{grad}(h) R(\lambda) .
\end{aligned}
$$

All terms are compact and integrable in norm. The second integrand in (9) is compact and integrable, too.

Proposition $2.13\left(H^{0}, F\right)$ is a Kasparov module over the pair of $C^{*}$-algebras $\left(C_{g}(M), A\right)$. The class $\left[H^{0}, F\right] \in \mathbf{K K}\left(C_{g}(M), A\right)$ is independent of the choice of $f$.

Proof: We have shown

$$
\begin{array}{rlll}
F & \in B\left(H^{0}\right)^{\text {odd }} & \text { Lemma } 2.8 & \\
F-F^{*} & \in K\left(H^{0}\right) & \text { Lemma } 2.9 & \\
F^{2}-1 & \in K\left(H^{0}\right) & \text { Lemma } 2.11 & \\
{[F, h]} & \in K\left(H^{0}\right) & \forall h \in C_{g}(M) \quad \text { Lemma } 2.12 & \\
F-F_{1} & \in K\left(H^{0}\right) & F, F_{1} \text { defined with } f, f_{1} \in C_{c}^{\infty}(M) & \text { Lemma } 2.10
\end{array}
$$

Recall the notation and geometric situation of the $\mathbf{K}$-theoretic relative index theorem.

\section{Theorem 2.14 (K-theoretic relative index theorem)}

$$
\left\{M_{1}\right\}+\left\{M_{2}\right\}-\left\{M_{3}\right\}-\left\{M_{4}\right\}=0
$$

Proof: The idea of the proof is as follows. The l.h.s. of $(10)$ is represented by $(\mathcal{H}, \mathcal{F})$, where

$$
\mathcal{H}=H_{1} \oplus H_{2} \oplus H_{3}^{o p} \oplus H_{4}^{o p}
$$


and

$$
\mathcal{F}=F_{1} \oplus F_{2} \oplus F_{3} \oplus F_{4} .
$$

We will construct an operator $X \in B(\mathcal{H})$ with $\operatorname{deg} X=1, X^{2}=1, X^{*}=X$, $[X, \mathcal{F}]:=X \mathcal{F}+\mathcal{F} X \in K(\mathcal{H})$. Thus

$$
[\mathcal{H}, \mathcal{F}] \in \operatorname{Im}\left(\beta^{*}\right): \mathbf{K K}\left(C^{0,1}, A\right) \rightarrow \mathbf{K K}(k, A)
$$

induced by the embedding $\beta: k \rightarrow C^{0,1}$. We show $\beta^{*}=0$ in Lemma 2.15.

We choose smooth cut-off functions $\chi_{W_{i}}, \chi_{V_{i}}, i=1,2$, with

$$
\begin{aligned}
\operatorname{supp} \chi_{W_{i}} & \subset W_{i} \cup U\left(N_{i}\right) \\
\operatorname{supp} \chi_{V_{i}} & \subset V_{i} \cup U\left(N_{i}\right) \\
\chi_{V_{i}}^{2}+\chi_{W_{i}}^{2} & =1 \\
\chi_{W_{1} \mid U\left(N_{1}\right)} & =\gamma^{*} \chi_{W_{2} \mid U\left(N_{2}\right)} \\
\chi_{V_{1} \mid U\left(N_{1}\right)} & =\gamma^{*} \chi_{V_{2} \mid U\left(N_{2}\right)} .
\end{aligned}
$$

Note that $L^{2}\left(W_{1} \cup U\left(N_{1}\right), E\right)$ is contained in $H_{1}$ as well as in $H_{3}$. We can define $a: H_{1} \rightarrow H_{3}$ by first multiplying by $\chi_{W_{1}}$ and then applying this identification of subspaces. Then $a^{*} a$ and $a a^{*}$ are simply the multiplications by $\chi_{W_{1}}^{2}$ on $H_{1}$ and $H_{3}$, respectively. We define $b: H_{1} \rightarrow H_{4}, c: H_{2} \rightarrow H_{3}$ and $d: H_{2} \rightarrow H_{4}$ analogously. Let $z \in B(\mathcal{H})$ be the $\mathbf{Z}_{2}$-grading and let

$$
X:=z\left(\begin{array}{cccc}
0 & 0 & -a^{*} & -b^{*} \\
0 & 0 & -c^{*} & d^{*} \\
a & c & 0 & 0 \\
b & -d & 0 & 0
\end{array}\right) \in B(\mathcal{H})
$$

Then $\operatorname{deg} X=1, X^{2}=1, X^{*}=X$. It remains to show

$$
X \mathcal{F}+\mathcal{F} X \in K(\mathcal{H}) \text {. }
$$

It is enough to verify the compactness of operators of the type

$$
a^{*} F_{3}-F_{1} a^{*} \in B\left(H^{3}, H^{1}\right) .
$$

Let

$$
h:=L^{2}\left(W_{1}, E_{1 \mid W_{1}}\right) \oplus L^{2}\left(V_{1}, E_{1 \mid V_{1}}\right) \oplus L^{2}\left(V_{2}, E_{2 \mid V_{2}}\right) .
$$

There are canonical embeddings $H_{1}, H_{3} \rightarrow h . F_{1}, F_{3}$ act naturally on $h$ after extension by zero to the subspace where they were not defined. Let $\chi:=\chi_{W_{1}}$ and $\rho \in C_{\mathrm{c}}^{\infty}\left(W_{1} \cup U\left(N_{1}\right)\right)$ be such that $\rho \chi=\chi$. The positive functions that were used to define $F_{i}$ are denoted by $f_{i}, i=1,2,3,4$. We can assume that $\chi f_{1}=\chi f_{3}$. We 
have

$$
\begin{aligned}
\chi F_{3}-F_{1} \chi & =\frac{2}{\pi} \int_{0}^{\infty}\left(\chi D_{3} R_{3}(\lambda)-D_{1} R_{1}(\lambda) \chi\right) d \lambda \\
& =\frac{2}{\pi} \int_{0}^{\infty}\left(\chi D_{3} R_{3}(\lambda)-D_{1} R_{1}(\lambda) \chi\left(D_{3}^{2}+f_{3}+\lambda^{2}\right) R_{3}(\lambda)\right) d \lambda \\
& =\frac{2}{\pi} \int_{0}^{\infty}\left(\chi D_{3} R_{3}(\lambda)-D_{1} R_{1}(\lambda) \chi\left(D_{1}^{2}+f_{1}+\lambda^{2}\right) \rho R_{3}(\lambda)\right) d \lambda \\
& =\frac{2}{\pi} \int_{0}^{\infty}\left(\chi D_{3} R_{3}(\lambda)-D_{1} \chi R_{3}(\lambda)-D_{1} R_{1}(\lambda)\left[\chi, D_{1}^{2}\right] \rho R_{3}(\lambda)\right) d \lambda \\
& =\frac{2}{\pi} \int_{0}^{\infty}\left(-\operatorname{grad}(\chi) R_{3}(\lambda)+D_{1} R_{1}(\lambda)\left(D_{1} \operatorname{grad}(\chi)+\operatorname{grad}(\chi) D_{1}\right) \rho R_{3}(\lambda)\right) d \lambda .
\end{aligned}
$$

The first term of the integrand is compact and integrable in the norm by Lemma 2.5. The second term can be written as

$D_{1}^{2} R_{1}(\lambda) \operatorname{grad}(\chi) R_{3}(\lambda)+D_{1} R_{1}(\lambda) \operatorname{grad}\left(f_{1}\right) R_{1}(\lambda) \operatorname{grad}(\chi) R_{3}(\lambda)+D_{1} R_{1}(\lambda) \operatorname{grad}(\chi) D_{3} R_{3}(\lambda)$ and every term is integrable and compact, too. This proves the desired compactness in (11). The theorem follows now from the following lemma.

Lemma 2.15 The embedding $\beta: k \rightarrow C^{0,1}$ induces the zero-map $\beta^{*}: \operatorname{KK}\left(C^{0,1}, A\right) \rightarrow$ $\mathbf{K K}(k, A)$.

Proof: Let $(H, F)$ represent an element in $\mathbf{K K}\left(C^{0,1}, A\right)$ and let $X \in C^{0,1}$ be the odd generator. Then $X \in B(H)$. Consider the operator homotopy

$$
F_{t}=\cos (t) F+\sin (t) X .
$$

We have $F_{0}=F, F_{\pi / 2}=X$ and hence $[H, F]=[H, X]=0 \in \mathbf{K K}(k, A)$.

\subsection{Cutting and pasting invariance}

Let $M^{n}$ be a closed Riemannian spin manifold and $V \rightarrow M$ be a flat bundle of projective modules over some real $C^{*}$ - algebra $A$. We consider the twisted Dirac operator $D: C^{\infty}(M, S) \rightarrow C^{\infty}(M, S)$ with $S:=E \otimes V$, where $E$ is the real Clifford bundle associated to the spin structure of $M$ admitting a right $C^{n, 0}$-action. Let $\{M\} \in \mathbf{K K}\left(\mathbf{R}, C^{n, 0} \otimes A\right)$ be the index of $D$.

Let $N \subset M$ be a compact two-sided hypersurface. We assume a tubular neighbourhood $U$ of $N$ with a product metric. Let

$$
\begin{array}{cccc}
\Gamma: & V_{\mid N} & \rightarrow & V_{\mid N} \\
& \downarrow & & \downarrow \\
\gamma: & N & \rightarrow & N
\end{array}
$$

be a commutative diagram of isomorphisms of all structures, in particular $\gamma$ is an isometry. Then we can cut $M$ at $N$ and glue together again using $\gamma$ in order to obtain $M_{1}$. Moreover, we can glue the bundle $S_{\mid M \backslash N}$ using $\Gamma$ to obtain a bundle $S_{1}$ over $M_{1}$ with Dirac operator $D_{1}$ giving $\left\{M_{1}\right\}$. 
Theorem 2.16 If the induced Riemannian metric on $N$ has positive scalar curvature, then $\{M\}=\left\{M_{1}\right\} \in \mathbf{K K}\left(\mathbf{R}, A \otimes C^{n, \mathbf{0}}\right)$.

Proof: We add to $M$ the manifold $\mathbf{R} \times N$, which has a Dirac operator induced from the restriction $S_{\mid N}$. This Dirac operator is invertible (Lemma 2.18) and hence $\{M\}=\{M\}+\{\mathbf{R} \times N\} . U$ has two boundary components $N_{ \pm}$. We partition $M$ into $M \backslash U$ and $U$, and $\mathbf{R} \times N$ into $(-\infty,-1] \times N \cup[1, \infty) \times N$ and $[-1,1] \times N$. There are identifications of the cutting hypersurfaces $i d: N_{-} \rightarrow\{-1\} \times N$ and $\gamma: N_{+} \rightarrow\{1\} \times N$. Analogously we identify the bundles using $\Gamma$ at the positive boundary part. We cut at these hypersurfaces and glue together, interchanging $U$ and $[-1,1] \times N$ and using the identifications given above, and obtain $M_{1} \cup \mathbf{R} \times N$ together with the corresponding Dirac operators. Again the cylinder does not contribute to the index. Applying the $\mathbf{K}$-theoretic relative index theorem we obtain $\{M\}=\left\{M_{1}\right\}$.

Since $\{M\},\left\{M_{1}\right\}$, do not depend on the choice of the Riemannian metric on $M, M_{1}$ it is enough to require that $N$ can carry a metric of positive scalar curvature such that $\gamma$ is an isometry. The compactness of $M$ is not important. It is enough to have invertibility of the Dirac operators at infinity. In the complex case a similar theorem is true for $A=\mathbf{C}$ without the assumption that $N$ carries a positive scalar curvature metric. This can be seen by the local index theorem. In the real case this theorem is not true even for $A=\mathbf{R}$ without the assumption as an example on $S^{1}$ shows. The author thanks K. Wojciechowski for pointing out an error in an earlier version of that paper.

\subsection{Cobordisms of positive scalar curvature metrics}

Fix a finitely generated group $\pi$. Any spin manifold $N$ with $\pi_{1}(N)=\pi$ gives rise to a $B:=B$ Spin $\times B \pi$-manifold (see [30]). The $B$ structure

$$
f: N \rightarrow B
$$

is given by the product of the classifying maps of the spin structure and of the universal cover of $N$. Consider the set $S_{n}(\pi)$ of tuples $(M, N, F, h)$ where $(M, N, F)$ is an $n$-dimensional $B$-manifold with $B$-structure $F$, with boundary $N=\partial M$ and $h$ is a positive scalar curvature metric on $N . S$ is a semigroup under disjoint union. Let $\sim$ be the equivalence relation given by $B$-bordism. A $B$-bordism of $(M, N, F, h)$ and $\left(M_{1}, N_{1}, F_{1}, h_{1}\right)$ consists of a $B$-manifold $\left(W, N, N_{1}, \Phi\right)$ with boundaries $\left(N, F_{\mid N}\right)$ and $\left(N_{1}, F_{1 \mid N_{1}}\right)$, a positive scalar curvature metric $g$ on $W$ which is a product metric near $\partial W$ and restricts to $h, h_{1}$ at $N, N_{1}$, and a $B$-manifold $(V, \Psi)$ with boundary $\left(M \cup_{N} W \cup_{N_{1}} M_{1},\left(F, \Phi, F_{1}\right)\right)$. Note that $R_{n}(\pi):=S_{n}(\pi) / \sim$ is a group. A similar group has been considered by $B$. Hajduk. It is a special case of a construction due to S. Stolz [29]. Let $C_{r}^{*}(\pi)$ be the reduced real group $C^{*}$-algebra of $\pi$.

Theorem 2.17 There is a canonical homomorphism $R_{n}(\pi) \rightarrow \mathbf{K K}_{n}\left(\mathbf{R}, C_{r}^{*}(\pi)\right.$ ). 
Proof: Let $(M, N, F, h) \in S_{n}(\pi)$. Choose a metric on $M$ such that it is a product metric near $N$ and restricts to $h$. Glue a metric cylinder $[0, \infty) \times N$ along the boundary of $M$ and obtain the complete manifold $\bar{M}$. Extend $F$ constantly over the cylinder. $F^{*} E \pi$ is a $\pi$-principal fibre bundle. Associate $C_{r}^{*}(\pi)$ and obtain a flat bundle $V$ with fibre $C_{r}^{*}(\pi)$ using the canonical action of $\pi$ on $C_{r}^{*}(\pi)$ from the left. Let $E$ be the real Clifford bundle with fibre $C^{n, 0}$ associated to the spin structure of $\bar{M}$ and form $S:=E \otimes V . S$ is a $\left(C_{r}^{*}(\pi) \otimes C^{n, 0}\right)-C^{*}$-Clifford bundle. Let $D$ be the associated Dirac operator. The scalar curvature is positive at infinity.

Lemma 2.18 $D$ is invertible at infinity

We can form $\{\bar{M}\} \in \mathbf{K K}\left(\mathbf{R}, C^{n, 0} \otimes C_{r}^{*}(\pi)\right)$. Clearly, the map associating the class $\{\bar{M}\}$ to $(M, N, F, h) \in S_{n}(\pi)$ is additive. We must show that it factors through $R_{n}(\pi)$. Let $(W, N, \Phi)$ be a $B$-manifold with boundary $\left(N, F_{\mid N}\right), g$ be a positive scalar curvature metric on $W$ which is a product metric near $\partial W$ and restricts to $h$ on $N$, and $(V, \Psi)$ be a $B$-manifold with boundary $\left(M \cup_{N} W,(F, \Phi)\right)$. Let $L:=\bar{M} \cup \bar{W}$ and $\tilde{L}:=W \cup_{N} M \cup \mathbf{R} \times N$. Then $\{L\}=\{\bar{M}\}$ and $\{\tilde{L}\}=\left\{W \cup_{N} M\right\}$ since, on the remaining components, there are positive scalar curvature metrics and the Dirac operators are invertible there. By the relative index theorem $\{L\}=\{\tilde{L}\}$. But $\{\tilde{L}\}=0$ since the Dirac operator on $W \cup_{N} M$ is zero-bordant. Hence $\{\bar{M}\}=0$. This proves the theorem. The idea for this construction was suggested by S. Stolz.

Proof of 2.18: (see also [28]). Because of the presence of the $C^{*}$-algebra we cannot apply standard theory. The main point is to verify that the image of $D^{2}+f: H^{2} \rightarrow$ $H^{0}$ is dense for a suitable $f \in C_{c}^{\infty}(\bar{M})$.

As a first step we show that $D+\lambda \epsilon$ is invertible for $\lambda \neq 0$. We realize $V$ as a direct summand of a bundle $W$ whose complexification is trivial. Consider the embedding of Hilbert- $C^{*}$-modules

$$
C_{r}^{*}(\pi) \rightarrow C_{r}^{*}(\pi) \otimes l_{\mathbf{R}}^{2}
$$

given by $f \rightarrow f \otimes e_{1}$, where $e_{1}$ is the first vector of an orthonormal base of $l_{\mathbf{R}}^{2}$. Let $W:=F^{*} E \pi \times_{\pi} C_{r}^{*}(\pi) \otimes l_{\mathbf{R}}^{2}$. Then $V$ is a direct summand in $W$. Let $W^{c}:=$ $W \otimes_{\mathbf{R}} \mathbf{C}$ be the complexification of $W$ and $V^{c}$ be the complexification of $V$. These complexifications carry real structures generally denoted by $\sigma$. By the result of [23] $W^{c}$ is trivial. Choose an isomorphism

$$
\Phi: W_{\mid M}^{c} \rightarrow M \times C_{r}^{*}(\pi) \otimes_{\mathbf{R}} l_{\mathbf{C}}^{2}=: U
$$

and extend it constantly over the cylindrical end. Since $M$ is compact, we can choose $\Phi$ smooth and $C^{1}$-bounded, i.e.

$$
\nabla^{W} \Phi-\Phi \nabla^{U} \in C_{b}\left(\bar{M}, T^{*} \bar{M} \otimes B\left(W^{c}, U^{c}\right)\right)
$$


Let $D_{U}$ and $D_{W}$ be the complex Dirac operators associated to $E^{c} \otimes U$ and $E^{c} \otimes W^{c}$. $D_{W}$ commutes with the real structure $\sigma$ of $E^{c} \otimes W^{c}$. We have $\left(D_{\alpha}+\lambda \epsilon\right)^{2}=D_{\alpha}^{2}+\lambda^{2}$ and for any $\phi \in H^{2}$ it holds

$$
\left\|\left(D_{\alpha}+\lambda \epsilon\right) \phi\right\|^{2}=\left\|D_{\alpha} \phi\right\|^{2}+\lambda^{2}\|\phi\|^{2} \geq \lambda^{2}\|\phi\|^{2},
$$

where $\alpha=U, W, E^{c}, \emptyset$. Since $U$ is trivial, $D_{U}+\lambda \epsilon$ is invertible for any $\lambda \neq 0$, $\left(D_{U}+\lambda \epsilon\right)^{-1}=\left(D_{E^{c}}+\lambda \epsilon\right)^{-1} \otimes 1_{U}$. We have a Neumann series

$$
\begin{aligned}
\left.\left(\Phi\left(D_{W}+\lambda \epsilon\right) \Phi^{-1}\right)\right)^{-1} & =\left(D_{U}+\lambda \epsilon+\left(\Phi D_{W} \Phi^{-1}-D_{U}\right)\right)^{-1} \\
& =\sum_{i=0}^{\infty}(-1)^{i}\left(\left(D_{U}+\lambda \epsilon\right)^{-1}\left(\Phi D_{W} \Phi^{-1}-D_{U}\right)\right)^{i}\left(D_{U}+\lambda \epsilon\right)^{-1}
\end{aligned}
$$

where the sum converges for $|\lambda|$ large enough because of (12) and (13). Thus, $D_{W}+\lambda \epsilon$, is invertible for large $\lambda$ and by the estimate (13) we can conclude that $D_{W}+\lambda \epsilon$ is invertible for any $\lambda \neq 0$ using again an argument with a Neumann series. Since $D_{W}+\lambda \epsilon$ commutes with the real structure, also its restriction to the real subspace is invertible. As $D+\lambda \epsilon$ is a direct summand it is invertible, too.

We show now that $D$ is invertible at infinity. $D^{2}+\lambda^{2}$ is invertible for $\lambda \neq 0$. Let $f \in C_{c}^{\infty}(M)$ be positive and such that $f+\tau / 4 \geq c>0$ where $\tau$ is the scalar curvature of $M$. We must show that $D^{2}+f$ is invertible too. We have the estimate

$$
\left\|\left(D^{2}+\lambda^{2}+f\right) \phi\right\|=\left\|\left(\Delta+\tau / 4+\lambda^{2}+f\right) \phi\right\| \geq c\|\phi\|
$$

uniformly in $\lambda$. Again using a Neumann series we can produce an inverse of $D^{2}+f \in B\left(H^{0}, H^{2}\right)$.

\section{Index theory for Callias type operators}

\subsection{Introduction}

Let $S \rightarrow M$ be a Clifford bundle (see Lawson/Michelsohn [21] for a definition) over a complete Riemannian manifold $M$. The associated Dirac operator with the domain $C_{c}^{\infty}(M, S)$ is essentially self-adjoint on the Hilbert space $H^{0}:=L^{2}(M, S)$ (Chernoff [13]). In order to apply the Fredholm index theory one has to assume $0 \notin \sigma_{e s s}(D)$ $\left(\sigma_{e s s}(D)\right.$ denotes the essential spectrum of $\left.D\right)$. This condition is not satisfied in general.

There is a way of obtaining another essentially self-adjoint operator $B:=D+\Phi$ by adding a suitable self-adjoint bundle endomorphism $\Phi \in C^{\infty}(M, \operatorname{End}(S))$ such that $0 \notin \sigma_{e s s}(B)$. $B$ is called a Callias-type operator (first examples were considered by Callias [12]). In this part of the paper we are concerned with the index theory of Callias-type operators. 
We will deal with the question of finding suitable $\Phi$ and with the computation of $i n d B$. The definition of the index of $B$ involves an auxiliary $\mathbf{Z}_{2}$-grading ( $B$ is self-adjoint). There is quite an effective and surprisingly easy method for computing ind $B$. All the information on the index of $B$ is contained in a tubular neighbourhood of a compact hypersurface $N \subset M$ cutting off the part where $\Phi$ is invertible. The computation can be reduced to an index computation on a compact manifold. In the example of Callias, $M=\mathbf{R}^{n}, n$ odd and ind $B$ is expressed as the index of a Dirac operator on a large sphere $S^{n-1} \subset \mathbf{R}^{n}$ twisted with a bundle of the positive eigenvectors of $\Phi$ on $S^{n-1}$. The result of Callias was generalized by Anghel [1],[2] to general odd-dimensional manifolds. Another example is Roe's index theorem for partitioned manifolds [25], which has a reformulation in terms of operators of Callias-type due to Higson [18].

All examples mentioned above start initially with a Dirac operator on an ungraded Clifford bundle $E \rightarrow M$. The grading enters later by forming matrices. They give non-trivial results on odd-dimensional manifolds.

There is an even-dimensional counterpart of the theory which starts with $\mathbf{Z}_{2}$ graded Dirac operators. The index problem for Callias-type operators on evendimensional manifolds has not been considered before.

In the present paper we adopt a more structural point of view (in contrast to [2], which focuses on the computation of the index). We will construct the endomorphisms $\Phi$ out of K-classes of the boundary $\partial_{h} M$ of a certain compactification $\bar{M}$ of $M$. This compactification was introduced by Higson [17] and described in [27]. $\partial_{h} M$ is called the Higson corona. It is the spectrum of the $C^{*}$-algebra $C_{g}(M) / C_{0}(M)$, where $C_{g}(M)$ is the $C^{*}$-subalgebra of $C(M)$ generated by the smooth bounded functions with vanishing gradient at infinity. Taking the index of $B:=D+\Phi$ leads to a homomorphism $u: \mathbf{K}^{0}\left(\partial_{h} M\right) \rightarrow \mathbf{Z}$ if $D$ is ungraded and $g: \mathbf{K}^{1}\left(\partial_{h} M\right) \rightarrow \mathbf{Z}$ if $D$ is graded.

The maps $u$ and $g$ are closely related with two other constructions, namely the spectral flow pairing $s: \mathbf{K}_{\mathbf{c}}^{1}(M) \rightarrow \mathbf{Z}$ (constructed with an ungraded operator) and the relative index pairing $r: \mathbf{K}_{c}^{0}(M) \rightarrow \mathbf{Z}$ (constructed with a graded Dirac operator). All these maps fit into the following commutative diagram which consists of the long exact sequence of complex $\mathbf{K}$-theory associated to the short exact sequence of $C^{*}$-algebras

$$
0 \rightarrow C_{0}(M) \rightarrow C_{g}(M) \rightarrow C\left(\partial_{h} M\right) \rightarrow 0
$$

completed with the maps $\mathrm{s}, \mathrm{r}, \mathrm{u}, \mathrm{g}$ to $\mathbf{Z}$. 


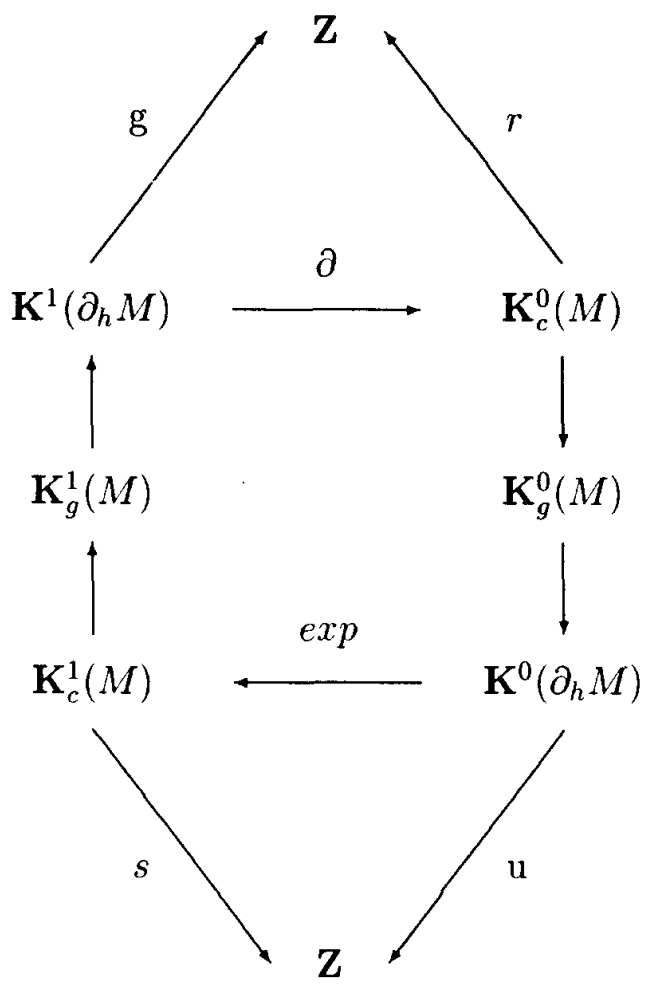

In order to save notations we have employed the identifications $\mathbf{K}_{g}^{*}(M):=\mathbf{K}_{*}\left(C_{g}(M)\right)$, $\mathbf{K}_{c}^{*}(M)=\mathbf{K}_{*}\left(C_{0}(M)\right)$ and $\mathbf{K}^{*}\left(\partial_{h} M\right):=\mathbf{K}_{*}\left(C\left(\partial_{h} M\right)\right)$.

It is very convenient to use the language of $\mathbf{K K}$-theory. Thus, we associate to $B:=D+\Phi$ a Kasparov module which represents a class $[M] \in \mathbf{K K}\left(C_{g}(M), \mathbf{C}\right)$ and the restriction $\{M\} \in \mathbf{K K}(\mathbf{C}, \mathrm{C})=\mathbf{Z}$ (here we use the convention that the symbol $M$ stands for all structures over $M$ ). We use the most general equivalence relation given by homotopy (see Blackadar [4], Jensen/Thomsen [20] for definitions). We will only need the definition of the KK-theory and the characterization (not the existence) of the intersection product by connections. Note, that $C_{g}(M)$ is not separable.

The Kasparov module associated to $B:=D+\Phi$ is an explicit example of a Kasparov intersection product $[\Phi] \otimes[D]$, where $[D]$ is a $\mathbf{K}$-homology class given by $D$ and $[\Phi]$ is a $\mathbf{K}$-class defined by $\Phi$. We will show this in the corresponding places.

We will compute the maps $g$ and $u$ in terms of the index of Dirac operators on certain compact manifolds. Our main computational tools are the deformation invariance of $\{M\}$, vanishing theorems for $\{M\}$ (e.g. if $\Phi$ is invertible or if $D$ is 
itself Fredholm) and the relative index theorem.

The idea of the index computation for a Callias-type operator is to use cutting and pasting and the vanishing theorems in order to reduce the problem to a cylinder over some compact hypersurface $N$. There, one can use separation of variables. This method was previously used by Roe [25], Higson [18] and Anghel [1], [2].

There is a close relation between Callias-type operators and the boundary map in $\mathbf{K}$-homology considered by Baum/Douglas/Taylor [3]. We can reprove the results of [3] modulo torsion. In a similar fashion the exotic index theory of Roe [27] is related to the index theory of Callias-type operators.

A large part of our methods also work in case of real operators and of operators being equivariant with respect to the action of some $C^{*}$-algebra $A$. The index of $B$ is then an element of $\mathbf{K}_{*}(A)$ (or of $\mathbf{K O}_{*}(A)$ in the real case). Difficulties arise in the case $A$ is infinite-dimensional. There are two critical points that have to be resolved. The first one is to verify that $D^{2}+1$ is invertible. This is not obvious (at least to the author) and equivalent to the density of the image of $D^{2}+1$. We prove a special case in Lemma 2.18. The second problem arises in our computations on the cylinder. Here, we explicitly identify kernels of two operators living on different spaces and want to conclude that their indices coincide. This is true if the kernels are complemented projective modules. However, in general this is not the case and one has to interpret the index as in Fomenko/Miščenko [24] (assuming $A$ ungraded to avoid even more complications). We hope to solve these problems (at least partially) in a forthcoming paper.

For the purpose of the present paper we consider examples of Callias-type operators being equivariant with respect to some real Clifford algebra $A:=C^{k, 0} . C^{k, l}$ is the real or complex Clifford algebra (depending in the context) generated by $e_{1}, \ldots, e_{k+l}$ subject to the relations

$$
e_{i} e_{j}+e_{j} e_{i}=\left\{\begin{array}{ccc}
0 & i & \neq j \\
-2 & i=j & \leq k \\
2 & l+k \geq i & =j>k
\end{array},\right.
$$

$e_{j}^{*}=-e_{j}, j \leq k$ and $e_{j}^{*}=e_{j}, l+k \geq j>k$.

In order to keep the paper in a reasonable size we have decided to be somewhat sketchy with the analytical details of the proofs. While, in the first section, we have to overcome serious analytical problems due to the presence of infinite-dimensional $C^{*}$-algebras, in the second part the arguments are much more standard. In several places we claim the compactness of a certain operator, that can be verified using the same methods as in the first section. We freely use product structures for Dirac operators near hypersurfaces, where we perform cutting and pasting. The details can be found e.g in Booß/Wojciechowski [5]. 


\subsection{The Higson corona and admissible endomorphisms}

Let $M$ be a complete Riemannian manifold with Dirac operator $D$ acting on sections of a complex Clifford bundle $S \rightarrow M$. We want to construct admissible $\Phi \in \Gamma(M, E n d(E))$ out of $\mathbf{K}$-classes living on some compactification $\bar{M}$ of $M$. An inspection of the constructions below shows that the largest compactification we can work with is the compactification by the Higson corona $\partial_{h} M$ (introduced by Higson [17], see also Roe [27]). K-classes living on smaller compactifications can be pulled back to $\partial_{h} M$ and we can apply our constructions to them, too.

Let $C(M)$ be the $C^{*}$-algebra of bounded continuous functions on $M$ with the sup-norm (we consider real or complex functions depending on the context). Let $C_{0}(M)$ be the closure of $C_{c}^{\infty}(M)$ in $C(M)$. Define

$$
C_{g}^{\infty}(M):=\left\{f \in C^{\infty}(M) \cap C(M)|| d f \mid \in C_{0}\left(M, T^{*} M\right)\right\}
$$

and let $C_{g}(M)$ be the closure of $C_{g}^{\infty}(M)$ in $C(M)$ (the index " " means compact support while " $0 "$ means vanishing at infinity). There is an exact sequence of $C^{*}$ algebras

$$
0 \rightarrow C_{0}(M) \rightarrow C_{g}(M) \rightarrow C\left(\partial_{h} M\right) \rightarrow 0,
$$

where $C\left(\partial_{h} M\right):=C_{g}(M) / C_{0}(M)$. The topological space $\partial_{h} M:=\operatorname{spec} C\left(\partial_{h} M\right)$ maximal ideal space of the $C^{*}$-algebra $C\left(\partial_{h} M\right) . \bar{M}:=\operatorname{spec} C_{g}(M)=M \cup \partial_{h} M$ is the smallest compactification to which all functions of $C_{g}(M)$ extend continuously.

The Higson corona is rather large. It maps to any smaller compactification defined by a subalgebra of $C_{g}(M)$, in particular to the one-point compactification and the end-compactification given by the closure of the algebra of functions being constant outside of a compact set.

Any element of $\mathbf{K}^{*}\left(\partial_{h} M\right):=\mathbf{K}_{*}\left(C\left(\partial_{h} M\right)\right)$ can be represented by a projection $(*=0)$ or a unitary $(*=1)$ in some matrix algebra $\operatorname{Mat}(l) \otimes C\left(\partial_{h} M\right)$ for some $l \geq 1$. Two such matrices represent the same element in $\mathbf{K}$-theory if they are homotopic as projections or unitaries in a possibly larger matrix algebra. We use the standard embeddings into the left upper corner

$$
\begin{gathered}
\operatorname{Mat}(l) \quad \mathcal{M a t}(l+1), \quad *=0 \\
U(l) \subset U(l+1), \quad *=1
\end{gathered}
$$

(often referred to as stabilization).

Definition 3.1 An endomorphism $\Phi \in C^{1}(M, E n d(S))$ is called admissible if

1. $\operatorname{deg} \Phi=1$ and $\Phi^{*}=\Phi$,

2. $\Phi D+D \Phi$ is bounded of zero order and

3. there is a compact set $K \subset M$ such that $\Phi D+D \Phi+\Phi^{2} \geq c>0$ on $M \backslash K$ for some constant $c$. 
A compact set $K \subset M$ with $\Phi^{2} \geq c>0$ on $M \backslash K$ is called an essential support of $\Phi$. Later we will show that all the information on the index of $B:=D+\Phi$ is contained in an essential support of $\Phi$. Note that by replacing $\Phi$ by $\Phi_{t}:=t \Phi$ one can have also $D \Phi_{t}+\Phi_{t} D+\Phi_{t}^{2}=t(D \Phi+\Phi D)+t^{2} \Phi^{2} \geq c>0$ outside of a given essential support for large $t . B$ is essentially self-adjoint with the domain $C_{c}^{\infty}(M, S)$ for admissible $\Phi$.

Lemma 3.2 If $\Phi \in C^{1}(M, E n d(S))$ is admissible, then $B:=D+\Phi$ is invertible at infinity (see (1)).

Proof: Let $K \subset M$ such that $\Phi D+D \Phi+\Phi^{2} \geq c>0$ on $M \backslash K$ for some constant $c$. Let $s \in C_{\mathbf{c}}^{\infty}(M)$ be a positive function such that $s \geq c$ on $K$. Then $B^{2}+\lambda s$ is self-adjoint and positive for large $\lambda$.

Let $s \in C_{c}^{\infty}(M), s \geq 0$, be such that $0 \leq\left(B^{2}+s\right) \in B\left(H^{0}, H^{2}\right)$ exists. Set $F:=B\left(B^{2}+s\right)^{-1 / 2}$. We call $\left(H^{0}, F\right)$ the Kasparov module associated to $B$ and it's class $\left[H^{0}, F\right] \in \mathbf{K K}\left(C_{g}(M), A\right)$ is independent of the choice of $s$ by Lemma 2.10, where $A$ is $\mathbf{C}$ or some $C^{k, 0}$ in the real examples ( $B$ is equivariant with respect to A).

Let $\Phi$ be an admissible endomorphism and $B:=D+\Phi$. Consider a continuous perturbation $B_{t}=D_{t}+\Phi_{t}$ of $B$ in $B\left(H^{1}, H^{0}\right)$ (where we define $H^{l}$ with $D_{0}$ ). Let $\left[M_{t}\right] \in \mathbf{K K}\left(C_{g}(M), A\right)$ be associated to $B_{t}$.

Lemma 3.3 $\left[M_{t}\right]=\left[M_{0}\right]$ for small $t$.

Proof: Let $s \in C_{c}^{\infty}(M), s \geq 0$, such that $\left(B^{2}+s\right)^{-1} \in B\left(H^{0}, H^{2}\right)$ exists. Then $\left(B_{t}^{2}+s\right)^{-1} \in B\left(H^{0}, H^{2}\right)$ for small $t$. The family $F_{t}$ is strictly continuous and the compact remainder terms $F_{t}-F_{t}^{*}, F_{t}^{2}-1$ and $\left[F_{t}, f\right], f \in C_{g}(M)$ are normcontinuous. This can be seen from the integral formulas for these operators given in the first section. Thus, $F_{t}$ defines a homotopy.

Corollary 3.4 Let $\left\{B_{t}\right\}_{t \in I}$ be a continuous family in $B\left(H^{1}, H^{0}\right)$ such that $B_{t}$ is invertible at infinity for any $t \in I$. Then $\left[M_{t}\right]=[M]$.

Proof: One finds a suitable finite partition of the interval $I$ and applies Lemma 3.3.

A continuous path $\Phi_{t} \in C(M, E n d(S))$ of admissible endomorphisms leads to a continuous family $B_{t} \in B\left(H^{1}, H^{0}\right)$. Further, a smooth deformation of $D$ over compact subsets does not affect the class $[M]$. Let $\{M\} \in \mathbf{K K}(k, A), k=\mathbf{C}, \mathbf{R}$, be the restriction of $[M]$.

Corollary 3.5 If $\Phi$ is admissible and has an empty essential support, then $\{M\}=$ 0 .

Proof: Consider the family $B_{t}:=D+t \Phi$. If $\Phi$ is admissible, so is $t \Phi$ for $t \geq 1$. Moreover, $B_{t}$ is invertible for large $t>0$. Hence $\{M\}=\left\{M_{t}\right\}=0$. 


\subsection{The odd-dimensional case}

\subsubsection{The map $u$}

Let $M$ be a complete Riemannian manifold and $E \rightarrow M$ be a (complex) Clifford bundle over $M$ with a Dirac operator $D_{E}$ that is ungraded. Let $[p] \in \mathbf{K}^{0}\left(\partial_{h} M\right)$ be represented by a projection $p \in C\left(\partial_{h} M\right) \otimes M a t(l)$ for some large $l$. We can find a "lift" $P \in C_{g}^{\infty}(M) \otimes M a t(l)$ of $p$ such that $P=P^{*}, P^{2}-P \in C_{0}^{\infty}(M) \otimes M a t(l)$ and $P\left(\bmod C_{0}(M) \otimes M a t(l)\right)$ represents $[p]$. Stabilization and homotopies of $p$ can be lifted to stabilization and homotopies of $P$. Form the $\mathbf{Z}_{2}$-graded Clifford bundle $S:=E \otimes\left(\mathbf{C}^{l} \oplus\left(\mathbf{C}^{l}\right)^{o p}\right)$ with the associated Dirac operator $D$ given by the matrix

$$
D:=\left(\begin{array}{cc}
0 & D_{E} \otimes 1 \\
D_{E} \otimes 1 & 0
\end{array}\right)
$$

Here $\mathbf{C}^{l}$ is trivially graded (i.e. all elements have degree 0 ) and $\left(\mathbf{C}^{l}\right)^{o p}$ consists of elements of degree 1 . Let

$$
\Phi:=\imath \otimes\left(\begin{array}{cc}
0 & 1-2 P \\
2 P-1 & 0
\end{array}\right) \in C^{1}(M, \operatorname{End}(S)) .
$$

Lemma 3.6 $\Phi$ is admissible.

Proof: We have $\operatorname{deg} \Phi=1$ and $\Phi=\Phi^{*}$. Moreover,

$$
D \Phi+\Phi D=2 \imath\left(\begin{array}{cc}
-\operatorname{grad} P & 0 \\
0 & \operatorname{grad} P
\end{array}\right)
$$

holds. $\operatorname{grad} P$ tends to zero at infinity while $\Phi^{2}$ tends to 1 . Thus, there is a compact set $K \subset M$ such that $D \Phi+\Phi D+\Phi^{2} \geq c>0$ on $M \backslash K$ for some constant $c$.

Let $\{M\} \in \mathbf{K K}_{0}(\mathbf{C}, \mathbf{C})=\mathbf{Z}$ be the class associated to the Callias-type operator

$$
B:=D+\Phi \text {. }
$$

Then $\{M\}$ only depends on the class of $p$ in $\mathbf{K}$-theory. In fact, two different choices of the lift $P$ can be deformed into each other such that we get a deformation of the corresponding admissible $\Phi$ 's. Stabilization results in forming a direct sum of $B$ with some invertible operator of Dirac type. Homotopies of $p$ inside finite matrices can be lifted to homotopies of the $P$ and thus of the $\Phi$ inside the admissible endomorphisms. All this does not affect the class $\{M\}$ (Lemma 3.4).

Definition 3.7 The map $u: \mathbf{K}^{0}\left(\partial_{h} M\right) \rightarrow \mathbf{Z}$ is defined by $u:[p] \rightarrow\{M\}$.

It is easy to see that $u$ is a group-homomorphism.

Since $D$ commutes with the action of $C^{0,1}$ given by

$$
\epsilon \rightarrow\left(\begin{array}{ll}
0 & 1 \\
1 & 0
\end{array}\right)
$$

where $\epsilon$ generates $C^{0,1}$, we have the following fact. 
Proposition 3.8 If there is a gap in the spectrum of $D_{E}$, then $u=0$.

Proof: Let $D$ and $\Phi$ be as in the construction above. $\Phi$ anticommutes with $\epsilon$. Let $\lambda \in \operatorname{res}(D) \cap \mathbf{R}$. Consider the two-parameter family

$$
B_{t, \tau}:=D-\tau \lambda \epsilon+t \Phi .
$$

We can deform $B_{1,0}$ to $B_{0,1}$ inside the operators of Callias-type that are invertible at infinity. Let first $\tau$ go from zero to one and then $t$ go from one to zero. $B_{0,1}$ and $B_{1,0}$ define the same $\mathbf{K K}$-klass. $B_{0,1}$ commutes with $\epsilon$ and, hence, defines the zero-element in KK-theory.

This fits well into the results of Roe and others stating that the odd index constructed out of an ungraded Dirac operator vanishes if there is a gap in the spectrum.

\subsubsection{Computation of $u$}

Let $N \subset M$ be a compact hypersurface cutting $M=M_{-} \cup_{N} M_{+}$such that $M_{-}$is compact and contains an essential support of $\Phi$. Deform the metric, the Clifford bundle structure and $\Phi$ in a neighbourhood of $N$ such that there is a product collar near $N$ and $\Phi$ is constant in normal direction. Let $M_{1}:=M_{-} \cup_{N}[0, \infty) \times N$. There is a Clifford bundle $S_{1} \rightarrow M_{1}$ respecting the product structure over the cylindrical end restricting to $S_{\mid M_{-}}$on $M_{-}$and $S_{1 \mid[0, \infty) \times N}=p r_{N}^{*} S_{\mid N}$. One can extend $-\Phi_{\mid M_{-}}$constantly along the cylinder obtaining $\Phi_{1}$. By scaling $\Phi$ and $\Phi_{1}$ we can assume that $\Phi_{1}$ is admissible. Form $M_{2}:=M \cup-M_{1}^{o p}$, where $o p$ means that we choose the opposite grading on the Clifford bundle over $M_{1}$ and - stands for redefining the Clifford bundle structure such that $X \in T M_{1}$ acts as $-X$ (note that we compress all structures over the manifolds into the symbols $\left.M, M_{1}, \ldots\right)$. Then $\left\{M_{2}\right\}=\{M\}-\left\{M_{1}\right\}$.

Now apply the relative index theorem. Cut the manifold $M_{2}$ at the two copies of $N$, i.e. $N \cup\{0\} \times N$, and glue them together interchanging the boundary components, thus obtaining $M_{3}:=M_{-} \cup_{N}-M_{-}^{o p} \cup M_{+} \cup_{N}-\left(\mathbf{R}_{+} \times N\right)^{o p}$. In order to glue the Clifford bundles we use an isomorphism

$$
\Gamma_{1}:=\left(\begin{array}{cc}
0 & n \\
n & 0
\end{array}\right)
$$

where $n$ is the Clifford multiplication by the unit normal vector field at $N$ pointing into the positive direction. It holds that $\Gamma_{1} \Phi=-\Phi \Gamma_{1}$ and the admissible endomorphisms glue nicely. By the relative index theorem $\left\{M_{2}\right\}=\left\{M_{3}\right\}$, but $\left\{M_{3}\right\}=0$. On the one hand, $\left\{M_{-} \cup_{N}-M_{-}^{o p}\right\}=0$ since this manifold is compact and, hence, the Dirac operator has a gap in the spectrum such that we can apply Proposition 3.8. On the other hand, an essential support of the admissible endomorphism over $M_{+} \cup_{N}-\left(\mathbf{R}_{+} \times N\right)^{o p}$ is empty. Thus $\left\{M_{+} \cup_{N}-\left(\mathbf{R}_{+} \times N\right)^{o p}\right\}=0$ by Corollary 3.5 
and, hence, $\{M\}=\left\{M_{1}\right\}$. We have replaced one half of the manifold by a cylinder. In the next step we do the same with the other half.

Consider $M_{4}:=M_{1} \cup-M_{1}$, where we choose the admissible endomorphism $\Phi_{4}$ as $-\Phi_{1}$ on the first component and as $\Phi_{1}$ on the second. Then $\left\{M_{4}\right\}=2\left\{M_{1}\right\}$. Now deform $\Phi_{4}$ on a compact set such that it is zero near the two copies $\{0\} \times N$ of $N$ in $M_{4}$. Cut $M_{4}$ at these two copies and glue them together again interchanging the boundary components, thus obtaining $M_{5}:=M_{-} \cup_{N}-M_{-} \cup M_{6}$ where $M_{6}=$ $\mathbf{R} \times N$. For glueing the Clifford bundle we use the even isomorphism of $S_{\left.\right|_{N}}$ given by multiplication by

$$
\Gamma_{2}:=\operatorname{diag}(n, n)
$$

By the relative index theorem $2\left\{M_{1}\right\}=\left\{M_{5}\right\}$, but $\left\{M_{-} \cup_{N}-M_{-}\right\}=0$ since this manifold is compact and therefore we can apply Proposition 3.8. Hence $2\{M\}=$ $\{\mathbf{R} \times N\}$. Thus we have reduced the index computation to a cylinder.

Let $\Phi_{c}$ be the admissible endomorphism over the cylinder. By a further deformation over compact sets we can assume that $\Phi_{c}=\psi \Phi_{\mid N}$, where $\psi$ is a smooth function on $\mathbf{R} \times N$ depending only on the first coordinate such that $\psi(r, n)=\operatorname{sign}(r)$ for $|r| \geq 2$ and $|\partial / \partial r \psi(r, n)| \leq 1 / 2$. Moreover, we can deform $\Phi_{\mid N}$ into an involution or, what is the same, $P_{\mid N}$ into a projection. This amounts to an index-preserving deformation of $\Phi_{c}$ by Lemma 3.4. Then

$$
\begin{aligned}
\{\mathbf{R} \times N\} & =\text { index }\left(n \frac{\partial}{\partial r}-\psi(r) \imath(1-2 P)+D_{N}\right) \\
& =\operatorname{index}\left(\frac{\partial}{\partial r}+\psi(r) n \imath(1-2 P)-n(1-2 P) D_{N}(1-2 P)\right)
\end{aligned}
$$

where $D_{N}$ is the Dirac operator on $S_{\mid N}$. We have

$(1-2 P) D_{N}(1-2 P)=(1-P) D_{N}(1-P)+P D_{N} P-P D_{N}(1-P)-(1-P) D_{N} P$

and $(1-P) D_{N} P$ and $P D_{N}(1-P)$ are of zero order. By an index-preserving deformation

$$
\{\mathbf{R} \times N\}=\operatorname{index} T \in \mathbf{K K}(\mathbf{C}, \mathbf{C})=\mathbf{Z}
$$

with

$$
T:=\left(\frac{\partial}{\partial r}+\psi(r) n \imath(1-2 P)-\left[n(1-P) D_{N}(1-P)+n P D_{N} P\right]\right) .
$$

By separation of variables (similar to the caluculation of Higson [18]) one obtains that the kernel of $T$ and $T^{*}$ consists of elements of the form

$$
\begin{aligned}
& f_{v}^{-}(r)=\exp \left(-\int_{0}^{r} \psi(s) \imath n(1-2 P)\right) v \\
& f_{v}^{+}(r)=\exp \left(\int_{0}^{r} \psi(s) \imath n(1-2 P)\right) v
\end{aligned}
$$


with $v \in \operatorname{kern}\left[(1-P) D_{N}(1-P)+P D_{N} P\right]$. The involution $\imath n(1-2 P)$ anticommutes with $n\left[(1-P) D_{N}(1-P)+P D_{N} P\right]$. We can split

$$
\operatorname{ker} n\left[(1-P) D_{N}(1-P)+P D_{N} P\right]=W^{+} \oplus W^{-}
$$

into the eigenspaces of $\imath n(1-2 P)$ to the eigenvalues $\pm 1 . f_{v}^{+}$is in $L^{2}$ iff $v \in W^{-}$ and $f_{v}^{-}$is in $L^{2}$ iff $v \in W^{+}$. Thus index $T=\operatorname{dim} W^{+}-\operatorname{dim} W^{-}$. Let $V_{-} \rightarrow N$ be the vector bundle over $N$ given by $P$ and $V_{+}$be the bundle given by $(1-P)$. Then $n\left[(1-P) D_{N}(1-P)+P D_{N} P\right]$ is the sum of the two twisted Dirac operators $D_{N, V_{+}}$ and $D_{N, V_{-}}$and $\operatorname{dim} W^{+}-\operatorname{dim} W^{-}=i n d_{2 n} D_{N, V_{+}}-i n d_{2 n} D_{N, V_{-}}$, where the grading is given by the involution $\imath n$. By cobordism invariance $i n d_{\imath n} D_{N, V_{+}}=-i n d_{\imath n} D_{N, V_{-}}$. Thus,

$$
u([p])=\{M\}=\frac{1}{2}\{\mathbf{R} \times N\}=\frac{1}{2} \text { index } T=\operatorname{ind}_{\imath n} D_{N, V_{+}} .
$$

Let us formulate this result as a theorem.

Theorem 3.9 Let $[p] \in \mathbf{K}^{0}\left(\partial_{h} M\right)$ and $P \in C_{g}^{\infty}(M) \otimes M a t(l)$ be a self-adjoint representative of $p$. Let $N \subset M$ be a compact hypersurface cutting $M=M_{-} \cup_{N} M_{+}$, where $M_{-}$is compact and $(1-2 P)^{2} \geq c>0$ on $M_{+}$for some constant $c$. Let $V_{+}$ be the sub-bundle of $N \times \mathbf{C}^{l}$ given by the eigenvalues $<\frac{1}{2}$ of $P_{\mid N}$. Then $u([p])=$ ind ${ }_{i n} D_{N, V_{+}}$, where $D_{N, V_{+}}$is the Dirac operator of $E_{\mid N} \otimes V_{+}$and the grading is given by the multiplication by in. $n$ is the unit normal vector field at $N$ pointing into the direction of $M_{+}$.

This theorem reproves and generalizes the theorems of Callias [12], Anghel [1] and was also proved in [2]. It contains the index theorem for partitioned manifolds of Roe [25] in the formulation of Higson [18] as a special case. In fact, a partitioned manifold $M$ is given by a complete Riemannian manifold $M$ together with a real function $\psi \in C^{\infty}(M)$ such that $|\psi|=1$ outside of a compact set and $\psi^{-1}(0)$ is a smooth compact hypersurface. Then $\psi$ induces an element $[(1+\psi) / 2] \in \mathbf{K}^{0}\left(\partial_{h} M\right)$. Let $N_{+} \cup N_{-}$be the boundary of a small tubular neighbourhood $M_{-}$of $N$ such that $\psi_{\mid N_{-}}<0$. By Theorem $3.9 u([\psi])=i n d_{\imath n} D_{N_{-}}=i n d_{\imath n} D_{N}$, where $n$ is the unit normal vector field pointing into the region of $M$, where $\psi$ is negative.

Since the index of a Dirac operator on an odd-dimensional manifold vanishes, we have $u=0$ if $M$ is even-dimensional.

\subsubsection{Interpretation as $\mathrm{KK}$-intersection product}

The operator $D:=D_{E} \otimes z X$ on $E \otimes C^{1,0}$ defines a class $[D] \in \mathbf{K K}\left(C_{0}(M) \otimes C^{1,0}, \mathbf{C}\right)$. We use the convention $(a \otimes b)(c \otimes d)=a c \otimes b d$ independent of the degree of the elements. This differs from the usual convention, where there is a sign $(-1)^{\operatorname{deg}(b) \operatorname{deg}(c)}$. $X$ is the odd generator of $C^{1,0}$ with $X^{2}=-1, X^{*}=-X$ and $z$ denotes the $\mathbf{Z}_{2^{-}}$ grading. Let $h:=L^{2}\left(M, E \otimes C^{1,0}\right)$ and $F_{2}:=D\left(D^{2}+1\right)^{-1 / 2} \in B(h)$. Then $\left[f \otimes X, F_{2}\right]=(f \otimes X) F_{2}+F_{2}(f \otimes X) \in K(h)$ for $f \in C_{0}(M)$. One can show this using an integral representation for $\left(D^{2}+1\right)^{-1 / 2}$ (similar as in Lemma 2.8) and the 
fact that $[f \otimes X, D]$ is an endomorphism of $E \otimes C^{1,0}$ vanishing at infinity. Moreover, $F_{2}^{*}=F_{2}$ and $f\left(F_{2}^{2}-1\right) \in K(h), \forall f \in C_{0}(M)$, and $\left(h, F_{2}\right)$ is a Kasparov module over $\left(C_{0}(M) \otimes C^{1,0}, \mathbf{C}\right)$ representing $[D]:=\left[h, F_{2}\right] \in \mathbf{K K}\left(C_{0}(M) \otimes C^{1,0}, \mathbf{C}\right)$.

Consider $L:=C_{0}(M) \otimes \mathbf{C}^{l} \otimes C^{1,0}$ as a graded $C_{0}(M) \otimes C^{1,0}$ - Hilbert- $C^{*}$-module. The endomorphism $\Phi:=\imath(1-2 P) \otimes X$ defines a bounded operator in $B(L)$. Moreover, $\Phi^{2}-1=4\left(P^{2}-P\right) \in C_{0}(M) \otimes M a t(l) \otimes C^{1,0}$ is in $K(L), \Phi^{*}=\Phi,[f, \Phi]=0$, $\forall f \in C_{g}(M)$ and $(L, \Phi)$ is a Kasparov module over the pair $\left(C_{g}(M), C_{0}(M) \otimes C^{1,0}\right)$ representing $[\Phi]:=[L, \Phi] \in \mathbf{K K}\left(C_{g}(M), C_{0}(M) \otimes C^{1,0}\right)$.

Let $\{\Phi\} \in \mathbf{K K}\left(\mathbf{C}, C_{0}(M) \otimes C^{1,0}\right)$ be the restriction of $[\Phi]$. Note that

$$
\mathbf{K K}\left(\mathbf{C}, C_{0}(M) \otimes C^{1,0}\right) \cong \mathbf{K}_{c}^{1}(M)
$$

(see Blackadar [4] 17.5.6.). Following [4] 17.5.4 the class $\{\Phi\}$ is represented by the self-adjoint unitary $\left(\bmod K\left(C_{0}(M) \otimes \mathbf{C}^{l}\right)\right) T:=1-2 P$. By [4] 17.5.6 the unitary $U:=\exp (2 \pi \imath P)$ corresponds to $\{\Phi\}$ under (15) and represents the class $\exp ([p]) \in \mathbf{K}_{c}^{1}(M)$.

Lemma 3.10 The class $\{\Phi\} \in \mathbf{K K}\left(\mathbf{C}, C_{0}(M) \otimes C^{1,0}\right)$ corresponds to $\exp ([p]) \in$ $\mathbf{K}_{c}^{1}(M)$ under the identification (15).

Let $B$ be the operator of Callias-type (14) and $[M] \in \mathbf{K K}\left(C_{g}(M), \mathbf{C}\right)$ be associated to $B$. Let $\tau_{C^{1,0}}: \mathbf{K K}\left(C_{g}(M), \mathbf{C}\right) \stackrel{\sim}{\rightarrow} \mathbf{K K}\left(C_{g}(M) \otimes C^{1,0}, C^{1,0}\right)$ be the isomorphism defined in Blackadar, [4] 17.8.5.

\section{Proposition 3.11}

$$
\tau_{C^{1,0}}[M]=[\Phi] \otimes_{C_{0}(M)}[D] \in \mathbf{K K}\left(C_{g}(M) \otimes C^{1,0}, C^{1,0}\right) .
$$

Proof: Consider $\tilde{H}:=L \otimes_{C_{0}(M)} h$. Then

$$
\tilde{H} \cong L^{2}(M, E) \otimes \mathbf{C}^{l} \otimes C^{1,0} \otimes C^{1,0} .
$$

The first $C^{1,0}$-factor comes from $L$ and carries the $C^{1,0}$-right action while the second $C^{1,0}$-factor comes from $h$ and carries the $C^{1,0}$ left action. We define the operator of Dirac type

$$
\tilde{B}:=D_{E} \otimes 1_{l} \otimes z \otimes z X+\imath(1-2 P) \otimes X \otimes 1
$$

Here $(1-2 P)$ acts on $L^{2}(M, E) \otimes \mathbf{C}^{l}$ and $z$ denotes the $\mathbf{Z}_{2}$-grading. $\tilde{B}$ is invertible at infinity and defines some $\tilde{F} \in B(\tilde{H})$. The same analysis as in the proof of Proposition 3.18 shows that $(\tilde{H}, \tilde{F})$ represents $[\Phi] \otimes_{C_{0}(M)}[D]$.

$[M]$ is represented by $(H, F)$, where $F$ was a bounded version of $B$. Moreover, $\tau_{C^{1,0}}[M]$ is represented by $\left(H \otimes C^{1,0}, F \otimes 1\right)$. The proof is finished by constructing a unitary $A: \tilde{H} \rightarrow H \otimes C^{1,0}$ intertwining with the left and right $C^{1,0}$-actions such that $(B \otimes 1) A=A \tilde{B}$. Then $(F \otimes 1) A-A \tilde{F} \in K\left(\tilde{H}, H \otimes C^{1,0}\right)$ follows.

We have $H=L^{2}(M, E) \otimes \mathbf{C}^{l} \otimes C^{1,0}$ and $B=D_{E} \otimes 1_{l} \otimes z X+\imath(1-2 P) \otimes X$. We define a graded $C^{1,0}$-bimodule isomorphism

$$
a: C^{1,0} \otimes C^{1,0} \rightarrow C^{1,0} \otimes C^{1,0}
$$

with respect to the actions 
- left multiplication by $c \in C^{1,0}$ on the 1.h.s: $c \rightarrow 1 \otimes c^{0}+z \otimes c^{1}$, where $c=c^{0}+c^{1}$ is the decomposition into homogeneous components

- right multiplication on the l.h.s.: $c \rightarrow c^{0} \otimes 1+c^{1} \otimes z$

- left multiplication on the r.h.s.: $c \rightarrow 1 \otimes c^{0}+z \otimes c^{1}$

- right multiplication on the r.h.s.: $c \rightarrow 1 \otimes c$.

Define $a$ as the left multiplication by

$$
\frac{1}{\sqrt{2}}(1 \otimes 1-X \otimes X z)
$$

and let

$$
A:=1_{E} \otimes 1_{l} \otimes a: \tilde{H} \rightarrow H \otimes C^{1,0} .
$$

An easy calculation shows that $A$ has the required properties.

\subsubsection{The spectral flow pairing $s$}

Let $M$ be a complete Riemannian manifold and $E \rightarrow M$ be an ungraded Clifford bundle over $M$. A cycle $[W] \in \mathbf{K}_{c}^{1}(M)$ is represented by a unitary matrix $W \in$ $C^{\infty}(M) \otimes M a t(l)$ with $W=1$ outside of a compact set $K \subset M$. Let $S:=E \otimes \mathbf{C}^{l}$ and $D$ be the associated Dirac operator. It is self-adjoint and defines a spectral projection $Q \in B\left(H^{0}\right)$ onto the positive spectral subspace of $D$. Consider $A:=(1-Q)+W Q$, where $W$ acts on $H^{0}$ as a unitary multiplication operator. It is shown in [11] that $A$ is a Fredholm operator.

Definition 3.12 The spectral flow pairing $s: \mathbf{K}_{c}^{1}(M) \rightarrow \mathbf{Z}$ is defined by $s([W]):=$ index $A$.

In fact, index $A$ depends only on the stable homotopy class of $W$ and is additive under direct sums. One can compute this index by reducing to a compact manifold containing $K$. If $M$ were compact, then $s([W])$ would be given by the spectral flow of a family between $D$ and $W^{*} D W$.

We will show the commutativity of the lower part of the diagram of the introduction. Let $\exp : \mathbf{K}^{0}\left(\partial_{h} M\right) \rightarrow \mathbf{K}_{c}^{1}(M)$ be the exponential map.

Theorem $3.13 u=s \circ \exp$

Proof: Let $[p] \in K^{0}\left(\partial_{h} M\right)$ be represented by a self-adjoint $P \in C_{g}^{\infty}(M) \otimes \operatorname{Mat}(l)$. We can choose $P$ such that $P$ is a projection outside of a compact set $K \subset M$. Then $\exp ([p])$ is represented by the unitary $W:=\exp (2 \pi \imath P)$. In fact, $W=1$ on $M \backslash K$. Choose a compact hypersurface $N \subset M$ which splits $M=M_{-} \cup_{N} M_{+}$ such that $K \subset M_{-}$. Let $I \times N$ be a tubular neighbourhood of $N$ in $M$. We can 
choose another $P_{1}$ representing $p$ such that $P_{1}=P$ on the plus-side of $I \times N$ and $P_{1}=0$ on the minus-side. Let $W_{1}=\exp \left(2 \pi \imath P_{1}\right)$. Then $W_{1}=1$ outside of the tubular neigbourhood of $N$ and $W_{1}$ represents the same class as $W$. Consider $P_{1}, W_{1}$ instead of $P, W$. As it has been shown in [11] one can compute $s([W])$ by reduction to $M_{1}:=S^{1} \times N$, a manifold which naturally containes our tubular neighbourhood $I \times N$. Deform the metric and the Clifford bundle structure near $N$ such that they respect the product structure of the tubular neighbourhood. Then glue $S_{I \times N}$ at the boundaries of $I \times N$, thus obtaining a Clifford bundle $S_{1} \rightarrow M_{1}$. Since $W=1$ on these boundaries, it extends to $W_{1}$ over $M_{1}$. Now form $A_{1}:=\left(1-Q_{1}\right)+W_{1} Q_{1}$, where $Q_{1}$ is the positive spectral projection of the Dirac operator on $S_{1}$. By the results of [11] index $A_{1}=$ index $A$ and index $A_{1}$ is the spectral flow of a family between $D_{1}$ and $W_{1}^{*} D_{1} W_{1}$. Since $W_{1}$ is the image of a projection $P_{1}:=P_{\mid 1 \times N}$ under the suspension map, this spectral flow equals $i n d_{n n} P_{1} D_{N} P_{1}$. A comparison with Theorem 3.9 finishes the proof.

The spectral flow pairing does not necessarily vanish if $D$ has a gap in the spectrum. It rather gives the spectral flow of the familiy between $D$ and $W^{*} D W$ in this gap [9]. If the class represented by $W$ is in the image of the exponential map and $D$ has a gap in the spectrum, then $s([W])=0$.

\subsection{The even-dimensional case}

\subsubsection{The construction of $g$}

Let $M$ be a complete Riemannian spin manifold and $E \rightarrow M$ be a $\mathbf{Z}_{2}$-graded Clifford bundle over $M$ with grading $z$. An element $[u] \in \mathbf{K}^{1}\left(\partial_{h} M\right)$ is represented by a unitary matrix $u \in C\left(\partial_{h} M\right) \otimes M a t(l)$ for some large $l$. Let $U \in C_{g}^{\infty}(M) \otimes M a t(l)$ be a "lift" of $u$ such that $U^{*} U-1 \in C_{0}(M) \otimes \operatorname{Mat}(l)$ and $U\left(\bmod C_{0}(M) \otimes M a t(l)\right)$ represents $[u]$. Stabilization and homotopies of $u$ can be lifted to stabilization and homotopies of $U$. Form the $\mathbf{Z}_{2}$-graded bundle $V:=M \times\left(\mathbf{C}^{l} \oplus\left(\mathbf{C}^{l}\right)^{o p}\right)$ and the graded tensor product $S:=E \otimes V . S$ is a Clifford bundle with Dirac operator $D$. Let

$$
\Phi:=z \otimes\left(\begin{array}{cc}
0 & U^{*} \\
U & 0
\end{array}\right)
$$

and

$$
B:=D+\Phi
$$

Lemma 3.14 $\Phi$ is admissible.

Proof: We have $\operatorname{deg} \Phi=1, \Phi^{*}=\Phi$ and

$$
D \Phi+\Phi D=z \otimes\left(\begin{array}{cc}
0 & -\operatorname{grad} U^{*} \\
-\operatorname{grad} U & 0
\end{array}\right)
$$


vanishes at infinity while $\Phi^{2}$ tends to 1 there. There is some compact set $K \subset M$ such that $D \Phi+\Phi D+\Phi^{2} \geq c>0$ on $M \backslash K$ for some constant $c$.

Let $\{M\} \in \mathbf{K K}(\mathbf{C}, \mathbf{C})=\mathbf{Z}$ be the class associated to the Callias-type operator (16.) It depends only on the stable homotopy class of $U$ and therefore only on $[u]$. Moreover, it is additive under the direct sums of $[u]$ 's.

Definition 3.15 The map $g: \mathbf{K}^{1}\left(\partial_{h} M\right) \rightarrow \mathbf{Z}$ is defined by $g([u]):=\{M\}$.

\subsubsection{Computation of $g$}

Let $N \subset M$ be a compact hypersurface cutting $M=M_{-} \cup_{N} M_{+}$such that $M_{-}$ is compact and contains an essential support of $\Phi$. We can deform $U$ to a unitary matrix on $M_{+}$using the polar decomposition. Thus, let us assume this. We further assume that (after deformation near $N$ ) the metric and the Clifford bundle structure near $N$ respect the product structure of a tubular neighbourhood $I \times N \subset M$ of $N$. Consider $M_{1}:=M \cup \mathbf{R} \times N$, where the Clifford bundle on $\mathbf{R} \times N$ comes from the restriction $S_{\mid N}$. We extend $\Phi_{\mid N}$ constantly along the $\mathbf{R}$-direction obtaining $\Phi_{1}$ over $M_{1}$. Then $\{M\}=\left\{M_{1}\right\}$ since an essential support of $\Phi_{1}$ over $\mathbf{R} \times N$ is empty. We apply now the relative index theorem. Cut at the two copies $N \cup\{0\} \times N$ and glue the pieces interchanging the boundary components, thus obtaining $M_{2}=$ $M_{-} \cup_{N} \mathbf{R}_{+} \times N \cup \mathbf{R}_{-} \times N \cup_{N} M_{+}$. The Clifford bundles are glued using the natural isomorphism. The admissible endomorphisms glue nicely to $\Phi_{2}$. Then $\{M\}=$ $\left\{M_{1}\right\}=\left\{M_{2}\right\}$. However, over $\mathbf{R}_{-} \times N \cup M_{+}$an essential support of $\Phi_{2}$ is empty and hence $\{M\}=\left\{M_{3}\right\}$, where $M_{3}:=M_{-} U_{N} \mathbf{R}_{+} \times N$. We have replaced half of the manifold by a cylinder.

Consider the matrix

$$
W:=\left(\begin{array}{cc}
U & 0 \\
0 & 1
\end{array}\right) \in C^{\infty}\left(M_{3}, \operatorname{End}\left(S_{3}\right)\right)
$$

$W$ is even, unitary and constant in the R-direction over the cylindrical end. Deform the connection $\nabla$ on $S_{3}$ near $\{1\} \times N$ such that it is $W \nabla W^{*}$ in a neighbourhood of $\{1\} \times N$ and deform $\Phi_{3}$ there such that

$$
\left(\begin{array}{ll}
0 & 1 \\
1 & 0
\end{array}\right)=W \Phi_{\mid N} W^{*}
$$

holds there. Now we cut $M_{-} \cup_{N} \mathbf{R}_{+} \times N$ at $\{1\} \times N \cup\{2\} \times N$ and glue the pieces together such that we obtain $M_{4}:=M_{-} \cup_{N}[0,1] \times N \cup_{N}[2, \infty] \times N \cup S^{1} \times N$. We use $W^{*}$ in order to glue the Clifford bundles

$$
W^{*}: S_{\mid\{1\} \times N} \rightarrow S_{\mid\{2\} \times N} .
$$

The admissible endomorphisms glue nicely to $\Phi_{4}$. By the relative index theorem $\{M\}=\left\{M_{4}\right\}$. Since $W \Phi_{\mid N} W^{*}$ can be extended invertibly over $M_{-} \cup_{N}[0,1] \times N$, 
we have $\{M\}=\left\{S^{1} \times N\right\}$. Since this manifold is compact, we can deform $\Phi_{4}$ to zero there. Let us formulate this result as a theorem.

Theorem 3.16 Let $[u] \in K^{-1}\left(\partial_{h} M\right)$ be represented by $U \in C_{g}^{\infty}(M) \otimes M a t(l)$. Let $N \subset M$ be a compact hypersurface of $M$ splitting $M=M_{-} \cup_{N} M_{+}$such that $M_{-}$ is compact and $U$ is invertible over $M_{+}$. Then $g([u])$ is given by the index of the twisted Dirac operator $D_{L}$ over $S^{1} \times N$ where $L \rightarrow S^{1} \times N$ is constructed from the trivial bundle $I \times N \times \mathrm{C}^{l}$ by glueing with

$$
U_{\mid N}^{*}:\{0\} \times N \times \mathbf{C}^{l} \rightarrow\{1\} \times N \times \mathbf{C}^{l} .
$$

$g([u])$ is also the spectral flow of a family between $D_{N}$ and $U_{\mid N}^{*} D_{N} U_{\mid N}$, where $D_{N}$ is a "half" of the Dirac operator on $S_{\mid N}$.

\subsubsection{Interpretation as KK-intersection product}

The operator $D_{E}$ on $E$ defines a class $[D] \in \mathbf{K K}\left(C_{0}(M), \mathbf{C}\right)$. Let $h:=L^{2}(M, E)$ and $F_{2}:=D_{E}\left(D_{E}^{2}+1\right)^{-1 / 2}$. Then $f\left(F_{2}^{2}-1\right) \in K(h),\left[F_{2}, f\right] \in K(h)$ for any $f \in C_{0}(M)$ and $F_{2}^{*}=F_{2}$. Thus $\left(h, F_{2}\right)$ is a Kasparov module over $\left(C_{0}(M), \mathbf{C}\right)$ and represents $[D]:=\left[h, F_{2}\right] \in \mathbf{K K}\left(C_{0}(M), \mathbf{C}\right)$.

Consider $L:=C_{0}(M) \otimes \mathbf{C}^{l} \otimes \mathrm{C}^{2}$ as a graded $C_{0}(M)$-Hilbert- $C^{*}$-module (the grading is induced by $\left.\mathbf{C}^{2}=\mathbf{C} \oplus(\mathbf{C})^{o p}\right)$. The endomorphism

$$
\Phi:=\left(\begin{array}{cc}
0 & U^{*} \\
U & 0
\end{array}\right)
$$

acts as a bounded operator in $B(L)$. Moreover,

$$
\Phi^{2}-1 \in C_{0}(M) \otimes \operatorname{Mat}(2 l) \subset K(L),
$$

$\Phi^{*}=\Phi,[f, \Phi]=0$ for all $f \in C_{g}(M)$ and $(L, \Phi)$ is a Kasparov module over the pair $\left(C_{g}(M), C_{0}(M)\right)$ representing $[\Phi]:=[L, \Phi] \in \mathbf{K K}\left(C_{g}(M), C_{0}(M)\right)$.

Let $\{\Phi\} \in \mathbf{K K}\left(\mathbf{C}, C_{0}(M)\right)$ be the restriction of $[\Phi]$. Note that

$$
\mathbf{K K}\left(\mathbf{C}, C_{0}(M)\right) \cong \mathbf{K}_{c}^{0}(M)
$$

(see Blackdar [4] 17.5.5). The unitary $U\left(\bmod K\left(C_{0}(M) \otimes \mathbf{C}^{l}\right)\right.$ ) defines an element $[U] \in \mathbf{K}_{1}\left(Q^{s} C_{0}(M)\right)$ where $Q^{s} C_{0}(M)$ is the stable outer multiplier algebra of $C_{0}(M)$. Then $\partial[U] \in \mathbf{K}_{0}\left(C_{0}(M)\right)=\mathbf{K}_{c}^{0}(M)$ is the element corresponding to $\{\Phi\}$ under the identification (17). This $\partial$ is associated to

$$
0 \rightarrow C_{0}(M) \otimes K \rightarrow M^{s} C_{0}(M) \rightarrow Q^{s} C_{0}(M) \rightarrow 0 .
$$

Since the index map $\partial$ is natural, we have

Lemma 3.17 The class $\{\Phi\} \in \mathbf{K K}\left(\mathbf{C}, C_{0}(M)\right)$ corresponds to $\partial[u] \in \mathbf{K}_{c}^{0}(M)$ under the identification (17). 
Let $B=D_{E} \otimes z+\Phi$ be the operator of Dirac type (16) (up to a non-important factor $z$ ) and $[M]:=[H, F] \in \mathbf{K K}\left(C_{g}(M), \mathbf{C}\right)$ be the class associated to $B$.

Proposition $3.18[M]=[\Phi] \otimes_{C_{0}(M)}[D]$

Proof: We have

$$
H=L \otimes_{C_{0}(M)} h=L^{2}(M, E) \otimes C^{l} \otimes C^{2} .
$$

We use the characterization of the intersection product in terms of connections (see Connes/Skandalis [14], Blackadar [4], Jensen/Thomsen [20]). It is enough to verify that $F$ is a $F_{2}$-connection and that $[F, \Phi] \geq 0(\bmod K(H))$. Let $f \otimes v \in L$, $f \in C_{0}(M), v \in C^{2 l}$. Then $T_{f \otimes v} \in B(h, H)$ is defined by

$$
T_{f \otimes v} \phi=f \phi \otimes v \quad \phi \in h .
$$

Its adjoint $T_{f \otimes v}^{*} \in B(H, h)$ is given by

$$
T_{f \otimes v}^{*}(\psi \otimes w)=f \psi<v, w>, \quad \psi \in L^{2}(M, E), \quad w \in C^{2 l} .
$$

By definition $F$ is and $F_{2}$-connection if for any $f \in C_{0}(M)$ and homogeneous $v \in C^{2 l}$

$$
\begin{aligned}
& T_{f \otimes v} F_{2}-(-1)^{\operatorname{deg}(v)} F T_{f \otimes v} \in K(h, H) \\
& F_{2} T_{f \otimes v}^{*}-(-1)^{\operatorname{deg}(v)} T_{f \otimes v}^{*} F \in K(H, h) .
\end{aligned}
$$

Let $\sim$ denote equality modulo compact operators. Then

$$
\begin{aligned}
F T_{f \otimes v} & \sim\left(B\left|B^{2}+s\right|^{-1 / 2} f(1 \otimes v)\right. \\
& \sim\left(D_{E} \otimes z\right)\left(D_{E}^{2} \otimes 1_{l}+1\right)^{-1 / 2} f(1 \otimes v)
\end{aligned}
$$

because of $f \in C_{0}(M)$. Since $\left[f, F_{2}\right] \in K(h)$, we obtain

$$
\begin{aligned}
(-1)^{\operatorname{deg}(v)} T_{f \otimes v} F_{2} & =(-1)^{\operatorname{deg}(v)} f D_{E}\left(D_{E}^{2}+1\right)^{-1 / 2} \otimes v \\
& \sim D_{E}\left(D_{E}^{2}+1\right)^{-1 / 2} f \otimes z v
\end{aligned}
$$

and

$$
F T_{f \otimes v}-(-1)^{\operatorname{deg}(v)} T_{f \otimes v} F_{2} \in K(h, H) .
$$

Next we consider

$$
F_{2} T_{f \otimes v}^{*}=D_{E}\left(D_{E}^{2}+1\right)^{-1 / 2} f \otimes v^{v},
$$

where $v^{v}:=<v, .>$ is the dual vector of $v$. It holds

$$
\begin{aligned}
(-1)^{\operatorname{deg}(v)} T_{f \otimes v}^{*} F & \sim(-1)^{\operatorname{deg}(v)}\left(f \otimes v^{v}\right) B\left|B^{2}+s\right|^{-1 / 2} \\
& \sim(-1)^{\operatorname{deg}(v)}\left(f \otimes v^{v}\right)\left(D_{E} \otimes z\right)\left(D^{2} \otimes 1_{l}+1\right)^{-1 / 2} \\
& \sim D_{E}\left(D_{E}^{2}+1\right)^{-1 / 2} f \otimes v^{v}
\end{aligned}
$$

Thus,

$$
F_{2} T_{f \otimes v}^{*}-(-1)^{\operatorname{deg}(v)} T_{f \otimes v} F \in K(H, h)
$$


and $F$ is an $F_{2}$-connection. We show the positivity condition.

$$
\begin{aligned}
{[\Phi, F] } & \sim\left[\Phi, B\left|B^{2}+s\right|^{-1 / 2}\right] \\
& =[\Phi,(D \otimes z+\Phi)]\left|B^{2}+s\right|^{-1 / 2}-B\left[\Phi,\left|B^{2}+s\right|^{-1 / 2}\right] \\
& \sim 2 \Phi^{2}\left|B^{2}+s\right|^{-1 / 2} \\
& \sim 2\left|B^{2}+s\right|^{-1 / 2} \\
& \geq 0 .
\end{aligned}
$$

We have employed that $\Phi$ has entries in $C_{g}^{\infty}(M)$ and that $\Phi^{2}-1 \in C_{0}(M) \otimes M a t(2 l)$.

The algebra $C_{g}(M)$ is not separable. Therefore, we use the strongest equivalence relation generated by homotopy. Other equivalence relations may give different $\mathbf{K K}$ groups. In the literature, in order to construct the product it is assumed in general that the algebra in the first place of the KK-group is separable. We do not use an abstract existence theorem, we rather write down an explicit module representing the product. In order to avoid non-separable algebras one could also consider the modules over $\mathbf{C}$ instead of $C_{g}(M)$ at the cost of losing some pieces of information.

\subsubsection{The relative index pairing $r$}

Let $M$ be a complete Riemannian manifold and $E \rightarrow M$ be a $\mathbf{Z}_{2^{-} \text {graded Clifford }}$ bundle over $M$. An element $\tau \in \mathbf{K}_{c}^{0}(M)$ is represented by a tuple $\left(V_{1}, V_{2}, W\right)$, where $V_{1}, V_{2}$ are vector bundles over $M$ and $W \in C^{\infty}\left(M, \operatorname{Hom}\left(V_{1}, V_{2}\right)\right)$ is invertible outside of a compact subset $K \subset M$. Let $N \subset M$ be a hypersurface cutting $M=M_{-} \cup_{N} M_{+}$ such that $M_{-}$is compact and contains $K$. Deform the metric and the Clifford bundle in order to have a product structure near $N$. Take $\tilde{M}=M_{-} \cup_{N}-M_{-}^{o p}$, where we glue the Clifford bundle obtaining $\tilde{E}$ using the odd morphism given by the multiplication by the unit normal vector field $n$ pointing into $M_{+}$. We glue $V_{1 \mid M_{-}}$with $V_{2 \mid-M_{-}^{\text {op }}}$ using the identification

$$
W_{\mid N}: V_{1 \mid M_{-}} \rightarrow V_{2 \mid-M_{-}^{o p}}
$$

obtaining $V \rightarrow \tilde{M}$. Let $\tilde{D}_{V}$ be the twisted Dirac operator on $\tilde{E} \otimes V$.

Definition 3.19 The relative index pairing $r: \mathbf{K}_{c}^{0}(M) \rightarrow \mathbf{Z}$ is defined by $r(\tau):=$ ind $\tilde{D}_{V}$.

This definition does not depend on the choice of the representative of $\tau$ and the hypersurface $N$ (for the latter see [16]).

We will show the commutativity of the upper part of the diagram of the introduction.

Theorem $3.20 r \circ \partial=g$. 
Proof: Let $[u] \in \mathbf{K}^{1}\left(\partial_{h} M\right)$ be represented by the unitary matrix $u \in C\left(\partial_{h} M\right) \otimes$ $M a t(l)$ and let $U \in C_{g}^{\infty}(M) \otimes M a t(l)$ be a "lift" of $u$. We can assume that $U$ is unitary outside of a compact set $K \subset M$. Then $\partial([u])$ is given by $\tau=\left[\left(M \times \mathbf{C}^{l}, M \times\right.\right.$ $\left.\mathrm{C}^{l}, U\right)$ ]. Let $N \subset M$ be a compact hypersurface cutting $M=M_{-} \cup_{N} M_{+}$such that $K \subset M_{-}$. Let $I \times N$ be a tubular neighbourhood of $N$. We can deform $U$ on $M_{-}$ such that $U_{M_{-} \backslash I \times N}=1$. Hence we can construct $r(\tau)$ as follows. We glue

$$
\tilde{M}=S^{1} \times N=I \times N \cup-(I \times N)^{o p} .
$$

We have to glue the trivial bundle $I \times N \times \mathrm{C}^{l}$ with the trivial bundle $-(I \times N)^{o p} \times \mathrm{C}^{l}$ using the identifications

$$
1:\{0\} \times N \times \mathbf{C}^{l} \rightarrow-(\{0\} \times N)^{o p} \times \mathbf{C}^{l}
$$

and

$$
U_{\mid\{1\} \times N}:\{1\} \times N \times \mathbf{C}^{l} \rightarrow-(\{1\} \times N)^{o p} \times \mathbf{C}^{l},
$$

thus obtaining $V \rightarrow S^{1} \times N$. Then $r(\tau)=$ ind $\tilde{D}_{V}$. A comparison with Theorem 3.16 gives the desired result.

\subsection{The boundary map in K-homology}

Let $\bar{M}$ be a compact Riemannian manifold with boundary $\partial \bar{M}=N$ and assume a metric product collar near $N$. We can make $\bar{M}$ complete by glueing a cylinder $\mathbf{R}_{+} \times N$ along $N$ and we obtain $M$. We choose a warped product metric $d r^{2}+f(r) g^{N}$ on the cylinder with $f(r) \rightarrow 0$ for $r \rightarrow \infty$. We can compactify $M$ by adjoining a copy of $N$ at infinity and obtain $M_{N}$. This compactification is associated with the algebra $C\left(M_{N}\right)$, which is the closure in $C(M)$ of the smooth bounded functions that are independent of the radial variable near infinity. Due to the choice of the metric $C\left(M_{N}\right) \subset C_{g}(M)$. Hence $M_{N}$ is a corona compactification (see Roe [27]) and all the results of the preceding subsections remain valid in this case. There is a canonical map $q: \partial_{h} M \rightarrow N$. One can lift $\mathbf{K}$-classes from $N$ to $\partial_{h} M$ and perform the constructions of Callias-type operators.

Let $E \rightarrow M$ be a graded $(*=0)$ or ungeraded $(*=1)$ Clifford bundle over $M$ with associated Dirac operator $D$. It defines a class $[D] \in \mathbf{K}_{*}^{c}(M)$. The exact sequence

$$
0 \rightarrow C_{0}(M) \rightarrow C\left(M_{N}\right) \rightarrow C(N) \rightarrow 0
$$

induces a long exact sequence in $\mathbf{K}$-homology

$$
\rightarrow \mathbf{K}_{*}\left(M_{N}\right) \rightarrow \mathbf{K}_{*}^{c}(M) \stackrel{\partial}{\rightarrow} \mathbf{K}_{*+1}(N) \rightarrow,
$$

where the grading of the $\mathbf{K}$-groups has values in $\mathbf{Z}_{2}$. The results of the preceding subsections can be used to characterize the boundary $\partial[D] \in \mathbf{K}_{*+1}(N)$ up to torsion. 
For every $x \in \mathbf{K}^{*+1}(N)$ we have already computed $<\partial x,[D]>\in \mathbf{Z}$. Thus, we know the pairings

$$
<x, \partial[D]>=<\partial x,[D]>\in \mathbf{Z}
$$

for all $x \in \mathbf{K}^{*+1}(N)$. This characterizes $\partial[D]$ up to torsion.

Let $E$ be ungraded and $*=1$. In Section 3.3.2 we have constructed a Dirac operator $D_{N}$ on $N$. It is associated to the Clifford bundle $E_{\mid N}$, which is now graded by the multiplication by $i n$, where $n$ is the outward-pointing unit normal vector field at $N$. By Theorem 3.9, Lemma 3.10 and Proposition 3.11 we have for any $[p] \in \mathbf{K}^{0}(N)$ represented by the projection $p \in \operatorname{Mat}(l) \otimes C(N)$ for some $l>0$

$$
<\partial[p],[D]>=u([p])=-i n d D_{N, V},
$$

where $V$ is the vector bundle on $N$ defined by $p$ and $D_{N, V}$ is the twisted Dirac operator. Thus for all $[p] \in \mathbf{K}^{0}(N)$

$$
<[p], \partial[D]>=-<[p],\left[D_{N}\right]>.
$$

Here $\left[D_{N}\right] \in \mathbf{K}_{0}(N)$ is the $\mathbf{K}$-homology class defined by the graded Dirac operator $D_{N}$ and we have used the fact that the pairing between $\mathbf{K}$-theory and $\mathbf{K}$-homology of $N$ is given by taking the index of twisted Dirac operators.

Theorem 3.21 $\partial[D]=-\left[D_{N}\right] \in \mathbf{K}_{0}(M)$ (mod torsion)

This in agrees with the result of Baum/Douglas/Taylor [3], Prop.5.1. The different sign in that reference is due to the use of the inner normal for grading the bundle $E_{\mid N}$.

Let now $E$ be graded and $*=0$. We have mentioned a Dirac operator $D_{N}$ acting on the ungraded bundle $E_{\mid N}^{+}$. From Theorem 3.16, Lemma 3.17 and Proposition 3.18 for any $[u] \in \mathbf{K}^{1}(N)$ represented by the unitary $u \in \operatorname{Mat}(l) \otimes C(N)$ it holds

$$
<\partial[u],[D]>=g([u])=s f\left\{D_{N}, u^{*} D_{N} u\right\},
$$

where $s f\left\{D_{N}, u^{*} D_{N} u\right\}$ is the spectral flow of a straight line between $D_{N}$ and $u^{*} D_{N} u$. Let $Q$ be the positive spectral projection of $D_{N}$. Then

$$
s f\left\{D_{N}, u^{*} D_{N} u\right\}=\operatorname{ind}(1-Q+u Q)=<[u],\left[D_{N}\right]>.
$$

$\left[D_{N}\right] \in \mathbf{K}_{1}(N)$ is the class defined by $D_{N}$ and we have used the fact that the spectral flow pairing is the pairing between the odd $\mathbf{K}$-theory and odd $\mathbf{K}$-homology of $N$.

Theorem 3.22 $\partial[D]=[D] \in \mathbf{K}_{1}(N)$ (mod torsion).

In order to compare this with the result of Baum/Douglas/Taylor we have to translate $\left[D_{N}\right]$ to the Cuntz-picture. Note that $\left[D_{N}\right]=[H, F] \in \mathbf{K} \mathbf{K}\left(C(N), C^{0,1}\right)$ with $H:=L^{2}(N, E) \otimes C^{0,1}$ and $F:=D_{N}\left(D_{N}^{2}+1\right)^{-1 / 2} \otimes X$, where $C(N)$ acts by 
left-multiplication on $H$. In the Cuntz-picture used in [3] (see Blackadar [4], 17.6.6) $\left[D_{N}\right]$ is given by the projection $(\bmod K)$

$$
P:=\frac{1}{2}\left(D_{N}\left(D_{N}^{2}+1\right)^{-1 / 2}+1\right)
$$

which commutes $(\bmod K)$ with the action of $C(N)$. However, $P=\mathcal{Q}(\bmod K)$, where $\mathcal{Q}$ is the Calderon projection of $D$, since both operators are pseudo-differential and have the same principal symbol. Thus $\partial[D]$ is also represented (mod torsion) by the Calderon projector of $D$ and this is in nice agreement with [3], Prop. 4.3.

Let $M$ be a complete Riemannian manifold and $\partial M$ be a reasonable corona in the sense of Roe, [27], 5.27, such that the transgression map $T: H^{*}(\partial M) \rightarrow H X^{*+1}(M)$ is defined. $H X^{*}(M)$ is the exotic cohomology introduced in [27]. Let $E \rightarrow M$ be a graded $(*=0)$ or ungraded $(*=1)$ Clifford bundle with Dirac operator $D$. Roe defined an index element $\operatorname{Ind}(D) \in \mathbf{K}_{*}\left(B_{H}\right)$, where $B_{H}$ is the algebra of locally tracable operators with bounded propagation on $H:=L^{2}(M, E)$ (see [27], 4.8 for $B_{H}$ and sections 4.3 and 4.6. for $\operatorname{Ind}(D)$ ). Let $\chi: H X^{*}(M) \rightarrow H C^{*}\left(B_{H}\right)$ be the character map from exotic to cyclic cohomology. Then, from the intermediate steps of the proof of Proposition [27], 5.2.9, we can obtain for any $x \in \mathbf{K}_{*+1}(\partial M)$

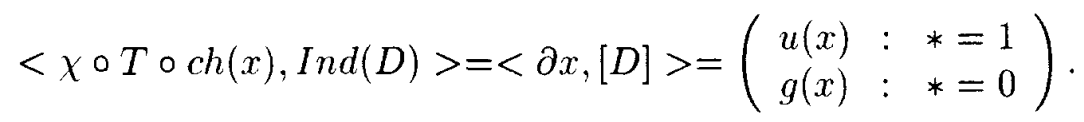

Thus, we have interpreted the pairing on the 1.h.s. of (18) as the index of a Calliastype operator. The author was informed by S. Hurder that G. Yu has a more satisfactory proof (unpublished) of the above fact solely using $\mathbf{K}$-theoretic arguments.

\subsection{Real Callias-type operators}

In this section we consider $\mathrm{KK}$-theory for real $C^{*}$-algebras and $C_{g}(M), C_{0}(M)$ etc. consist of real functions.

Let $M$ be a complete Riemannian manifold and $E \rightarrow M$ be a real $\mathbf{Z}_{2}$-graded Clifford bundle over $M$ admitting a right action of the real Clifford algebra $C^{n, 0}$. Let $z$ be the grading operator. The Dirac operator $D_{E}$ is $C^{n, 0}$-equivariant and provides an element $[D] \in \mathbf{K K}\left(C_{0}(M), C^{n, 0}\right)$. $[D]$ is represented by $(H, F)$ with $H:=L^{2}(M, E)$ and $F:=D_{E}\left(D_{E}^{2}+1\right)^{-1 / 2}$. For later purpose it is useful to employ $[D]_{k, n-k} \in \mathbf{K K}\left(C_{0}(M) \otimes C^{0, k}, C^{n-k, 0}\right)$. For $k \leq n$ there is a map

$$
\beta_{k}: \mathbf{K K}\left(C_{0}(M), C^{n, 0}\right) \rightarrow \mathbf{K K}\left(C_{0}(M) \otimes C^{0, k}, C^{n-k, 0}\right) .
$$

Let $(H, F)$ represent some element $[H, F] \in \mathbf{K K}\left(C_{0}(M), C^{n, 0}\right)$. Then $\beta_{k}[H, F]$ is represented by the same $(H, F)$. The $C_{0}(M) \otimes C^{0, k}$-left action on $H$ is given by 
$f \otimes e \rightarrow f z e$, where $e \in \mathbf{R}^{k}, f \in C_{0}(M)$ and $z$ is the $\mathbf{Z}_{2}$-grading. We assume that $\mathbf{R}^{k} \subset \mathbf{R}^{n}$ generates $C^{0, k}$ as well as $C^{k, 0} \subset C^{n, 0}$ and $e$ acts by the right multiplication. Let $\mathbf{R}^{n-k}=\left(\mathbf{R}^{k}\right)^{\perp} \subset \mathbf{R}^{n}$ generate $C^{n-k, 0}$, then $C^{n-k, 0}$ commutes with $f z e, e \in \mathbf{R}^{k}$. If we restrict the right multiplication to $C^{n-k, 0}$, then we get a Kasparov module $(H, F)$ over the pair $\left(C_{0}(M) \otimes C^{0, k}, C^{n-k, 0}\right)$. Set $[D]_{k, n-k}:=\beta_{k}[D]$.

We want to pair $[D]_{k, n-k}$ with certain elements $[\Phi] \in \mathbf{K K}\left(C_{g}(M), C_{0}(M) \otimes C^{0, k}\right)$. We consider a $[\Phi]$ represented by a matrix $\Phi \in \operatorname{Mat}(l) \otimes C_{g}^{\infty}(M) \otimes C^{0, k}$ for some $l>0$ with the properties $\Phi^{*}=\Phi, \operatorname{deg} \Phi=1$ and that $\Phi^{2}-1$ vanishes at infinity. We construct an operator of Callias-type representing

$$
[M]=[\Phi] \otimes_{C_{0}(M) \otimes C^{0, k}}[D]_{k, n-k} \in \mathbf{K K}\left(C_{g}(M), C^{n-k, 0}\right) .
$$

Form the graded tensor product $S:=\left(C^{0, k}\right)^{\oplus l} \otimes_{C^{0, k}} E=\mathbf{C}^{l} \otimes E$. This is a real $\mathbf{Z}_{2^{-}}$ graded Clifford bundle with right action of $C^{n-k, 0}$. Let $D=1_{l} \otimes D_{E}$ be the Dirac operator of $S$ and form $B:=D+\Phi$. The Callias-type operator $B$ is equivariant with respect to $C^{n-k, 0}$.

Lemma $3.23 \Phi$ is admissible.

The same analysis as in Section 3.4.3 shows

Proposition 3.24

$$
[\Phi] \otimes_{C_{0}(M) \otimes C^{0, k}}[D]_{k, n-k}=[M] \in \mathbf{K K}\left(C_{g}(M), C^{n-k, 0}\right)
$$

is the class associated to $B$.

Let $\{M\} \in \mathbf{K K}\left(\mathbf{R}, C^{0, n-k}\right)$ be the restriction of $[M]$.

In order to compute $\{M\}$ we will make a special choice of $\Phi$. Let $f \in C\left(\partial_{h} M\right) \otimes$ $\mathbf{R}^{k}$ be given such that $f^{2}=-1$, i.e. $f$ has values in the unit sphere. Let $F \in$ $C_{g}^{\infty}(M) \otimes \mathbf{R}^{k}$ be a lift of $f$ and set $\Phi:=z F$.

We can assume that 0 is a regular value of $F$. Then $N:=F^{-1}(0)$ is a compact manifold of dimension $n-k$. We use $F$ in order to identify a small neighbourhood of $N$ with $D^{k} \times N$, where $D^{k}$ is the unit disk in $\mathbf{R}^{k}$. After deformation of the metric we can assume that $F$ is an isometry on this neighbourhood. Let $\tilde{F}$ be a deformation of $F$ in a neighbourhood of $N$ such that $\tilde{F}(x, n)=x /|x|$ in a neighbourhood of $S^{k-1} \times N=\partial\left(D^{k} \times N\right)$. Let $M_{1}:=\mathbf{R}^{k} \times N$ with $F_{1}(x, n)=\tilde{F}(x, n)$ on $D^{k} \times N$ and $x /|x|$ for $|x| \geq 1$. $M_{1}$ has a Clifford bundle $S_{1}$ induced from $S$ respecting the product structure. Let $\Phi_{1}:=-z F_{1}$ and $M_{2}:=M \cup-M_{1}^{o p}$. Cut $M_{2}$ at the two copies of $S^{k-1} \times N$ and glue the pieces together interchanging the boundary components, thus obtaining $M_{3}=M_{4} \cup M_{5}$ with

$$
M_{4}:=M \backslash D^{k} \times N \cup_{S^{k-1} \times N}-\left(R^{k} \backslash D^{k} \times N\right)^{o p}
$$

and

$$
M_{5}:=D^{k} \times N \cup_{S^{k-1} \times N}-\left(D^{k} \times N\right)^{o p} .
$$


We glue the Clifford bundles using the odd morphism given by left multiplication with the unit normal vector field at the boundary $S^{k-1} \times N$. By the relative index theorem $\left\{M_{2}\right\}=\left\{M_{3}\right\}$, but $\left\{M_{3}\right\}=0$. In fact, $M_{5}$ is compact and $\Phi_{5}$ can be deformed to zero. The Dirac operator itself, without perturbation, is equivariant with respect to a larger Clifford algebra. On $M_{4}$ an essential support of the admissible endomorphism is empty. Hence $\{M\}=\left\{M_{1}\right\}$. Thus, we have reduced the problem to a product $M_{1}=\mathbf{R}^{k} \times N$, where $\Phi_{1}(x, n)=z x /|x|$ for $|x| \geq 1$.

Let $M:=M_{1}$ and $\Phi:=\Phi_{1}$ for a moment. The Clifford bundle $S \rightarrow \mathbf{R}^{k} \times N$ is the pull back of a bundle $S^{k}$ over $N$ admitting a right action of $C^{n, 0}$ and a left action of $C^{k, 0}$ (which commutes with the action of the Clifford algebra bundle of $N$ in a graded way). This left action is used to define the Clifford multiplication with $T \mathbf{R}^{k}$ on $p r_{N}^{*} S^{k}$ in order to obtain the Clifford bundle $S$ over $\mathbf{R}^{k} \times N$. We want to use induction in $k$. In order to employ the product structure $\mathbf{R}^{k}=\mathbf{R} \times \mathbf{R}^{k-1}$ we have to deform $\Phi$ such that it respects this product structure. For $1 / 2>\epsilon>0$ let $\psi \in C^{\infty}(\mathbf{R})$ be such that $\psi(r)=\operatorname{sign}(r)$ for $|r| \geq 2 / \epsilon, \psi(r) \in[-1,1]$ for $|r| \leq 2 / \epsilon$ and $\left|\partial_{r} \psi\right| \leq 2 \epsilon$. Define $\Phi_{1}(x, n):=z\left(\psi\left(x^{1}\right), \ldots, \psi\left(x^{k}\right)\right) \in \mathbf{R}^{k}$.

Lemma 3.25 $\Phi_{1}$ is admissible.

Proof: We have $\operatorname{deg} \Phi_{1}=1$ and $\Phi_{1}^{*}=\Phi_{1}$. Moreover, $\Phi_{1} D+D \Phi_{1}+\Phi_{1}^{2}=$ $-z \operatorname{grad}\left(z \Phi_{1}\right)+\Phi_{1}^{2}$. But $\left|\operatorname{grad}\left(z \Phi_{1}\right)\right| \leq 2 k \epsilon$ and for $|x| \geq 2 k / \epsilon$ we have $\Phi_{1}^{2} \geq 1$.

Let $\left\{M_{1}\right\}$ be the class associated to $B_{1}:=D+\Phi_{1}$.

Lemma 3.26 For $\epsilon>0$ small enough it holds that $\{M\}=\left\{M_{1}\right\}$.

Proof: We can deform $\Phi$ to $\Phi_{1}$ inside the admissible endomorphisms. Let $\Phi_{t}=$ $t \Phi+(1-t) \Phi_{1}$. Then, for $|x| \geq 2 k / \epsilon$,

$$
\begin{aligned}
& \Phi_{t} D+D \Phi_{t}+\Phi_{t}^{2} \\
= & -t z \operatorname{grad}(z \Phi)-(1-t) z \operatorname{grad}\left(z \Phi_{1}\right)+t^{2} \Phi^{2}+(1-t)^{2} \sum_{i=1}^{k} \psi\left(x^{i}\right)^{2} \\
& \quad+2 t(1-t) \frac{1}{|x|} \sum_{i=1}^{k} x^{i} \psi\left(x^{i}\right) \\
\geq & t^{2}+(1-t)^{2}-t|\operatorname{grad}(z \Phi)|-2(1-t) k \epsilon \\
\geq & 1 / 8
\end{aligned}
$$

for $\epsilon$ small enough.

Let now $\mathbf{R}^{k}=\mathbf{R} \times \mathbf{R}^{k-1}$ and $n$ be the unit normal vector in the $\mathbf{R}$-direction. $n$ generates $C^{1,0}$ and we can split $S^{k}=S^{k-1} \otimes C^{1,0}$ by the following procedure. Let $e:=z l r$, where $l$ is the action of $n$ from the left and $r$ is the action of $n$ from the right. $e$ is an even involution of $S^{k}$. Let $S^{k-1}$ be the positive eigenspace of $e$. Then $S^{k-1}$ admits a $C^{n-1,0}$-action from the right, where $C^{n-1}$, is generated by $\mathbf{R}^{\perp} \subset \mathbf{R}^{n}$, 
is a Clifford bundle over $N$ and admits a $C^{k-1,0}$ action from the left commuting in the graded sense with the action of the Clifford algebra bundle of $N$. In fact, the generators of $C^{k-1,0}$ acting from the left and of $C^{n-1,0}$ acting from the right commute with the involution $e$.

For $0 \leq i \leq k$ let $M^{(i)}:=\mathbf{R}^{i} \times N$ with the Clifford bundle induced from $S^{i}$. Construct the admissible endomorphism $\Phi_{1}^{(i)}$ as above and form $\left\{M^{(i)}\right\}$.

Proposition 3.27 $\left\{M^{(i)}\right\}=\left\{M^{(i-1)}\right\}$

Proof: We identify $\operatorname{ker}\left(D^{(i)}+\Phi^{(i)}\right)$ with $\operatorname{ker}\left(D^{(i-1)}+\Phi^{(i-1)}\right)$ as a $C^{n-k, 0}$-module. With respect to $S^{i}=S^{i-1} \otimes C^{1,0}$ we have

$$
D^{(i)}+\Phi^{(i)}=l \frac{\partial}{\partial x^{i}}+z r \psi\left(x^{i}\right)+\left[D^{(i-1)}+\Phi^{(i-1)}\right] \otimes 1 .
$$

Multiplying by $-l$ we obtain

$$
A:=\frac{\partial}{\partial x^{i}}+z \operatorname{lr} \psi\left(x^{i}\right)-l\left[D^{(i-1)}+\Phi^{(i-1)}\right] \otimes 1 .
$$

$A$ respects the splitting

$L^{2}\left(\mathbf{R}^{i} \times N, S^{i}\right)=L^{2}(\mathbf{R}) \otimes k \operatorname{er}\left(\left[D^{(i-1)}+\Phi^{(i-1)}\right] \otimes 1\right) \oplus L^{2}(\mathbf{R}) \otimes \operatorname{ker}\left(\left[D^{(i-1)}+\Phi^{(i-1)}\right] \otimes 1\right)^{\perp}$

and is invertible on the. second summand. The elements of ker $A$ are of the form

$$
f_{v}\left(x^{i}\right)=\exp \left(-z l r \int_{0}^{x^{i}} \psi(t) d t\right) v
$$

From the $L^{2}$-condition we see that $\operatorname{ker} A$ is the 1 -eigenspace of $z \operatorname{lr}$ in $\operatorname{ker}\left(\left[D^{(i-1)}+\right.\right.$ $\left.\left.\Phi^{(i-1)}\right] \otimes 1\right)$, but this is exactly $\operatorname{ker}\left(D^{(i-1)}+\Phi^{(i-1)}\right)$. All identifications we have made respect the structure of graded $C^{n-k, 0}$-modules. This proves the Proposition since the class $\{M\}$ is determined by the kernel of the Callias-type operator as a graded $C^{n-k, 0}$-module.

We come now back to the general situation. Let $M$ be a complete Riemannian manifold and $S \rightarrow M$ be a real $\mathbf{Z}_{2}$-graded real Clifford bundle over $M$ admitting a right $C^{n, 0}$-action with associated Dirac operator $D$. Let $F \in C_{g}^{\infty}(M) \otimes \mathbf{R}^{k}$ for $0<k \leq n$ as above and $N:=F^{-1}(0)$. We have constructed a Clifford bundle $S^{0} \rightarrow N$ with Dirac operator $D_{N}^{(0)}$ admitting a right $C^{n-k, 0}$-action. Putting the above results together we have

Theorem 3.28 $\{M\}=$ ind $D_{N}^{(0)}$.

We will apply this result to obstructions against the existence of positive scalar curvature metrics. Let $\left(M^{n}, g\right)$ be a complete Riemannian spin manifold of dimension $n$. There is a natural real Clifford bundle $S \rightarrow M$ admitting a $C^{n, 0}$-action 
$S:=P_{S p i n} \times_{S p i n(n)} C^{n, 0}$. Let $F \in C_{g}^{\infty}(M) \otimes \mathbf{R}^{k}, n \geq k \geq 1$, with $|F| \rightarrow 1$ at infinity. Assume that $0 \in \mathbf{R}^{k}$ is a regular value of $F$ and define $N:=F^{-1}(0)$. Then $N$ is a compact spin manifold and $S^{0}$ is the natural Clifford bundle associated to the spin structure of $N$. In this case ind $D_{N}^{(0)}=\alpha(N)$, where $\alpha(N)$ is the alpha invariant of $N$ (see e.g. Hitchin [19] and Lawson/Michelson [21]). Let $D$ be the Dirac operator of $S$. Let $\tau$ denote the scalar curvature of $M$. By the Weitzenböck formula, if $\tau$ is uniformly positive at infinity, then $D^{2}=\Delta+\tau / 4 D$ is invertible at infinity. In this case we can deform the admissible endomorphism to zero without changing the index. However, $D$ is equivariant with respect to a larger Clifford algebra $C^{n, 0}$ and hence the class of its kernel vanishes. It follows $\alpha(N)=0$. Recall that two Riemannian metrics $g_{0}, g_{1}$ are called quasi-isometric if there is a constant $C<\infty$ such that

$$
\frac{1}{C} g_{0} \leq g_{1} \leq C g_{0}
$$

Since the conditions on $F$ only depend on the quasi-isometry class of the Riemannian metric on $M$, we obtain

Corollary 3.29 Let $\left(M^{n}, g\right)$ be a complete Riemannian spin manifold and $F \in$ $C_{g}^{\infty}(M) \otimes \mathbf{R}^{k}$ be such that $|F| \rightarrow 1$ at infinity and 0 is a regular value. If $\alpha\left(F^{-1}(0)\right) \neq$ 0 , then there is no metric in the quasi-isometry class of $g$ that has uniform positive scalar curvature at infinity.

This generalizes similar results of Roe [27] to dimensions $n-k=1,2(\bmod 8)$, where the index has values in $\mathbf{Z}_{2}$. A special case is:

Corollary 3.30 If $\alpha(N) \neq 0$, then there is no metric with uniform positive scalar curvature at infinity on the product $\mathbf{R}^{k} \times N$ for any $k$ in the quasi-isometry class of the product metric.

Corollary 3.31 If $\alpha(N) \neq 0$, then there is no metric of non-negative scalar curvature $\tau$ on $\mathbf{R}^{k} \times N$ with lower bound $\tau(x, n) \geq \frac{c}{|x|+1}$ for some $c>0$ in the quasiisometry class of the product.

Proof: Assume that $M:=\mathbf{R}^{k} \times N$ has a metric with non-negative scalar curvature in the quasi-isometry class of the product satisfying $\tau(x, n) \geq \frac{c}{|x|+1}$ for some constant $c>0$. Consider $B_{t}:=D+t z F$ with $F \in C^{\infty}\left(M, \mathbf{R}^{k}\right)$ depending only on the first coordinate and satisfying $F^{-1}(0)=\{0\} \times N$ and $F(x, n)=x /|x|$ for $|x|>1$. Then $B_{t}^{2}=\Delta+\tau / 4-z t \operatorname{grad} F+t^{2} F^{2}$. But $|\operatorname{grad} F(x, n)| \leq \frac{c_{1}}{|x|+1}$ for some $c_{1}>0$. Thus

$$
\tau / 4-z t \operatorname{grad} F+t^{2} F^{2} \geq \frac{c / 4-c_{1} t}{|x|+1}+t^{2} F^{2}
$$

If we chose $t<\frac{c}{4 c_{1}}$, then $B_{t}$ is positive and hence invertible. It follows that $\{M\}=\left\{M_{t}\right\}=0$ and $\alpha(N)=0$. 

Let $M$ again be a complete Riemannian spin manifold with non-negative scalar curvature. Let $F \in C^{\infty}(M) \otimes \mathbf{R}^{k}$, having 0 as a regular value and being constant of unit length outside of a tubular neighbourhood $U$ of $N=F^{-1}(0)$. Assume that the scalar curvature is positive on $U$.

Corollary $3.32 \alpha(N)=0$

Proof: Consider $B_{t}=D+t z F$ for small $t>0$. $B_{t}$ is invertible at infinity. Thus, by Theorem 3.28 and the deformation invariance $\alpha(N)=\left\{M_{1}\right\}=\left\{M_{t}\right\}$. Then $B_{t}^{2}=\Delta+\tau / 4-z t \operatorname{grad}(F)+t^{2}|F|^{2}$. If $t$ is small enough, then $\tau / 4-z \operatorname{tgrad}(F) \geq 0$ and $B_{t}$ is invertible, but then $\left\{M_{t}\right\}=0$.

This sort of argument was used in Lesch [22] in order to prove

Corollary 3.33 ([22]) If $\alpha(N) \neq 0$, then there is no complete Riemannian metric on $\mathbf{R} \times N$ that is positive on some section $\{a\} \times N \subset \mathbf{R} \times N$.

\section{References}

[1] N. Anghel. $L^{2}$-index formulae for perturbed Dirac operators. Commun.Math.Phys., 128(1990),77-97.

[2] N. Anghel. On the index of Callias-type operators. Geom.Funct.Anal., $3(5)(1993), 432-438$.

[3] P. Baum, R. G. Douglas, and M. E. Taylor. Cycles and relative cycles in analytic K-homology. J.Diff.Geom., 30(1989),761-804.

[4] B. Blackadar. K-Theory for Operator Algebras. Math.Sci.Res.Inst.Publ. No. 5 Springer, New York, 1986.

[5] B. B. Booß-Bavnbek and K. P. Wojciechowski. Elliptic Boundary Problems for Dirac Operators. Birkhäuser, Boston-Basel-Berlin, 1993.

[6] N. V. Borisov, W. Müller, and R.Schrader. Relative index theory and supersymmetric scattering theory. Commun.Math.Phys., 114(1988),475-513.

[7] J. Brüning and H. Moscovici. $L^{2}$-index for certain Dirac-Schrödinger operators. Duke. Math. J., 66(2)(1992),311-336.

[8] U. Bunke. Dirac Operatoren auf offenen Mannigfaltigkeiten. PhD thesis, ErnstMoritz-Arndt-Universität Greifswald, 1991.

[9] U. Bunke. On the spectral flow of families of Dirac operators with constant symbol. To appear in: Math.Nachrichten, 1993.

[10] U. Bunke. Relative index theory. J.Funct.Anal., 105(1)(1992),63-76. 
[11] U. Bunke and T. Hirschmann. The index of the scattering operator on the positive spectral subspace. Commun.Math.Phys., 148(1992),478-502.

[12] C. Callias. Axial anomalies and index theorems on open spaces. Commun.Math.Phys., 62(1978),213-234.

[13] P. Chernoff. Essential selfadjointness of powers of generators of hyperbolic equations. J.Funct.Anal., 12(1973),401-414.

[14] A. Connes and G. Skandalis. The longitudinal index theorem for foliations. Publ.RIMS, Kyoto Univ., 20(1984),1139-1183.

[15] H. Donnelly. Essential spectrum and heat kernel. J.Funct.Anal., $75(2)(1987), 362-381$.

[16] M. Gromov and H. B. Lawson. Positive scalar curvature and the Dirac operator on complete Riemannian manifolds. Publ.Math IHES, 58(1983),295-408.

[17] N. Higson. K-homology and operators on non-compact manifolds. Preprint, 1988.

[18] N. Higson. A note on the cobordism invariance of the index. Topology, $30(3)(1991), 439-443$.

[19] N. Hitchin. Harmonic spinors. Adv.in Math., 14:(1974),1-55.

[20] K. K. Jensen and K. Thomsen. Elements of KK-theory. Birkhäuser BostonBasel-Berlin, 1991.

[21] H. B. Lawson and M. L. Michelsohn. Spin Geometry. Princeton University Press, 1989.

[22] M. Lesch. Deficiency indices, cobordism invariance of the Clifford index and positive scalar curvature. Preprint Nr. 262, Augsburg, 1992.

[23] J. A. Mingo. K-theory and multipliers of stable $C^{*}$-algebras. Trans.AMS, $299(1)(1987), 387-411$.

[24] A. S. Miščenko and A. T. Fomenko. The index of elliptic operators over $C^{*}$ algebras. Izv.Akad.Nauk SSSR,Ser.Math., 43(1979),831-859.

[25] J. Roe. Partitioning non-compact manifolds and the dual Toeplitz problem. In Operator Algebras and Applications, pp. 187-228. Cambridge Univ. Press, 1989.

[26] J. Roe. A note on the relative index theorem. Quart.J.Math. Oxford, $42(2)(1991), 365-373$. 
[27] J. Roe. Coarse cohomology and index theory on complete Riemannian manifolds. Memoirs of the AMS, 104(497), 1993.

[28] J. Rosenberg. $C^{*}$-algebras, positive scalar curvature, and the Novikov conjecture. Publ.Math.I.H.E.S., 58(1983),197-212.

[29] S. Stolz. Concordance classes of positive scalar curvature metrics. In preparation, 1993.

[30] R. E. Stong. Notes on Cobordism Theory. Princeton University Press, 1968. 
